\title{
Measurement of isolated photon production in deep-inelastic scattering at HERA
}

\section{The H1 Collaboration}

F.D. Aaron ${ }^{5, a}$, A. Aktas ${ }^{11}$, C. Alexa ${ }^{5}$, V. Andreev ${ }^{25}$, B. Antunovic ${ }^{26}$, S. Aplin ${ }^{11}$, A. Asmone ${ }^{33}$, A. Astvatsatourov ${ }^{4}$, S. Backovic ${ }^{30}$, A. Baghdasaryan ${ }^{38}$, P. Baranov ${ }^{25}$, E. Barrelet ${ }^{29}$, W. Bartel ${ }^{11}$, S. Baudrand ${ }^{27}$, M. Beckingham ${ }^{11}$,

K. Begzsuren ${ }^{35}$, O. Behnke ${ }^{14}$, O. Behrendt ${ }^{8}$, A. Belousov ${ }^{25}$, N. Berger ${ }^{40}$, J.C. Bizot ${ }^{27}$, M.-O. Boenig ${ }^{8}$, V. Boudry ${ }^{28}$, I. Bozovic-Jelisavcic ${ }^{2}$, J. Bracinik ${ }^{26}$, G. Brandt ${ }^{14}$, M. Brinkmann ${ }^{11}$, V. Brisson ${ }^{27}$, D. Bruncko ${ }^{16}$, F.W. Büsser ${ }^{12}$,

A. Bunyatyan ${ }^{13,38}$, G. Buschhorn ${ }^{26}$, L. Bystritskaya ${ }^{24}$, A.J. Campbell ${ }^{11}$, K.B. Cantun Avila ${ }^{22}$, F. Cassol-Brunner ${ }^{21}$,

K. Cerny ${ }^{32}$, V. Cerny ${ }^{16, b}$, V. Chekelian ${ }^{26}$, A. Cholewa ${ }^{11}$, J.G. Contreras ${ }^{22}$, J.A. Coughlan ${ }^{6}$, G. Cozzika ${ }^{10}$,

J. Cvach ${ }^{31}$, J.B. Dainton ${ }^{18}$, K. Daum ${ }^{37, c}$, M. Deak ${ }^{11}$, Y. de Boer ${ }^{24}$, B. Delcourt ${ }^{27}$, M. Del Degan ${ }^{40}$, J. Delvax ${ }^{4}$,

A. De Roeck ${ }^{11, d}$, E.A. De Wolf ${ }^{4}$ C. Diaconu ${ }^{21, e}$, V. Dodonov ${ }^{13}$, A. Dubak ${ }^{30, f}$, G. Eckerlin ${ }^{11}$, V. Efremenko ${ }^{24}$,

S. Egli ${ }^{36}$, R. Eichler ${ }^{36}$, F. Eisele ${ }^{14}$, A. Eliseev ${ }^{25}$, E. Elsen ${ }^{11}$, S. Essenov ${ }^{24}$, A. Falkiewicz ${ }^{7}$, P.J.W. Faulkner ${ }^{3}$,

L. Favart ${ }^{4}$, A. Fedotov ${ }^{24}$, R. Felst ${ }^{11}$, J. Feltesse ${ }^{10, g}$, J. Ferencei ${ }^{16}$, L. Finke ${ }^{11}$, M. Fleischer ${ }^{11}$, A. Fomenko ${ }^{25}$,

G. Franke ${ }^{11}$, T. Frisson ${ }^{28}$, E. Gabathuler ${ }^{18}$, J. Gayler ${ }^{11}$, S. Ghazaryan ${ }^{38}$, S. Ginzburgskaya ${ }^{24}$, A. Glazov ${ }^{11}$,

I. Glushkov ${ }^{39}$, L. Goerlich ${ }^{7}$, M. Goettlich ${ }^{12}$, N. Gogitidze ${ }^{25}$, S. Gorbounov ${ }^{39}$, M. Gouzevitch ${ }^{28}$, C. Grab ${ }^{40}$,

T. Greenshaw ${ }^{18}$, B.R. Grell ${ }^{11}$, G. Grindhammer ${ }^{26}$, S. Habib ${ }^{12, h}$, D. Haidt ${ }^{11}$, M. Hansson ${ }^{20}$, G. Heinzelmann ${ }^{12}$,

C. Helebrant ${ }^{11}$, R.C.W. Henderson ${ }^{17}$, H. Henschel ${ }^{39}$, G. Herrera ${ }^{23}$, M. Hildebrandt ${ }^{36}$, K.H. Hiller ${ }^{39}$, D. Hoffmann ${ }^{21}$,

R. Horisberger ${ }^{36}$, A. Hovhannisyan ${ }^{38}$, T. Hreus ${ }^{4, i}$, M. Jacquet ${ }^{27}$, M.E. Janssen ${ }^{11}$, X. Janssen ${ }^{4}$, V. Jemanov ${ }^{12}$,

L. Jönsson ${ }^{20}$, D.P. Johnson ${ }^{4}$, A.W. Jung ${ }^{15}$, H. Jung ${ }^{11}$, M. Kapichine ${ }^{9}$, J. Katzy ${ }^{11}$, I.R. Kenyon ${ }^{3}$, C. Kiesling ${ }^{26}$,

M. Klein ${ }^{18}$, C. Kleinwort ${ }^{11}$, T. Klimkovich ${ }^{11}$, T. Kluge ${ }^{11}$, A. Knutsson ${ }^{11}$, V. Korbel ${ }^{11}$, P. Kostka ${ }^{39}$, M. Kraemer ${ }^{11}$,

K. Krastev ${ }^{11}$, J. Kretzschmar ${ }^{39}$, A. Kropivnitskaya ${ }^{24}$, K. Krüger ${ }^{15}$, M.P.J. Landon ${ }^{19}$, W. Lange ${ }^{39}$,

G. Laštovička-Medin ${ }^{30}$, P. Laycock ${ }^{18}$, A. Lebedev ${ }^{25}$, G. Leibenguth $^{40}$, V. Lendermann ${ }^{15}$, S. Levonian ${ }^{11}$, G. Li $^{27}$,

L. Lindfeld ${ }^{41}$, K. Lipka ${ }^{12}$, A. Liptaj ${ }^{26}$, B. List ${ }^{12}$, J. List ${ }^{11}$, N. Loktionova ${ }^{25}$, R. Lopez-Fernandez ${ }^{23}$, V. Lubimov ${ }^{24}$,

A.-I. Lucaci-Timoce ${ }^{11}$, L. Lytkin ${ }^{13}$, A. Makankine ${ }^{9}$, E. Malinovski ${ }^{25}$, P. Marage ${ }^{4}$, L. Marti ${ }^{11}$, M. Martisikova ${ }^{11}$,

H.-U. Martyn ${ }^{1}$, S.J. Maxfield ${ }^{18}$, A. Mehta ${ }^{18}$, K. Meier ${ }^{15}$, A.B. Meyer ${ }^{11}$, H. Meyer ${ }^{11}$, H. Meyer ${ }^{37}$, J. Meyer ${ }^{11}$,

V. Michels ${ }^{11}$, S. Mikocki ${ }^{7}$, I. Milcewicz-Mika ${ }^{7}$, A. Mohamed ${ }^{18}$, F. Moreau ${ }^{28}$, A. Morozov ${ }^{9}$, J.V. Morris ${ }^{6}$,

M.U. Mozer ${ }^{4}$, K. Müller ${ }^{41}$, P. Murín ${ }^{16, i}$, K. Nankov ${ }^{34}$, B. Naroska ${ }^{12}$, T. Naumann ${ }^{39}$, P.R. Newman ${ }^{3}$,

C. Niebuhr ${ }^{11}$, A. Nikiforov ${ }^{11}$, G. Nowak ${ }^{7}$, K. Nowak ${ }^{41}$, M. Nozicka ${ }^{39}$, R. Oganezov ${ }^{38}$, B. Olivier ${ }^{26}$, J.E. Olsson ${ }^{11}$,

S. Osman ${ }^{20}$, D. Ozerov ${ }^{24}$, V. Palichik ${ }^{9}$, I. Panagoulias ${ }^{11, j, k}$, M. Pandurovic ${ }^{2}$, T. Papadopoulou ${ }^{11, j, k}$,

C. Pascaud ${ }^{27}$, G.D. Patel ${ }^{18}$, H. Peng ${ }^{11}$, E. Perez ${ }^{10, d}$, D. Perez-Astudillo ${ }^{22}$, A. Perieanu ${ }^{11}$, A. Petrukhin ${ }^{24}$,

I. Picuric ${ }^{30}$, S. Piec ${ }^{39}$, D. Pitzl ${ }^{11}$, R. Plačakytè ${ }^{11}$, R. Polifka ${ }^{32}$, B. Povh ${ }^{13}$, T. Preda ${ }^{5}$, P. Prideaux ${ }^{18}$, V. Radescu ${ }^{11}$,

A.J. Rahmat ${ }^{18}$, N. Raicevic ${ }^{30}$, T. Ravdandorj ${ }^{35}$, P. Reimer $^{31}$, C. Risler ${ }^{11}$, E. Rizvi ${ }^{19}$, P. Robmann ${ }^{41}$, B. Roland ${ }^{4}$,

R. Roosen $^{4}$, A. Rostovtsev ${ }^{24}$, Z. Rurikova ${ }^{11}$, S. Rusakov ${ }^{25}$, D. Salek ${ }^{32}$, F. Salvaire ${ }^{11}$, D.P.C. Sankey ${ }^{6}$, M. Sauter ${ }^{40}$,

E. Sauvan ${ }^{21}$, S. Schmidt ${ }^{11}$, S. Schmitt ${ }^{11}$, C. Schmitz ${ }^{41}$, L. Schoeffel ${ }^{10}$, A. Schöning ${ }^{40}$, H.-C. Schultz-Coulon ${ }^{15}$,

F. Sefkow ${ }^{11}$, R.N. Shaw-West ${ }^{3}$, I. Sheviakov ${ }^{25}$, L.N. Shtarkov ${ }^{25}$, T. Sloan ${ }^{17}$, I. Smiljanic ${ }^{2}$, P. Smirnov ${ }^{25}$,

Y. Soloviev ${ }^{25}$, D. South ${ }^{8}$, V. Spaskov ${ }^{9}$, A. Specka ${ }^{28}$, Z. Staykova ${ }^{11}$, M. Steder ${ }^{11}$, B. Stella ${ }^{33}$, J. Stiewe ${ }^{15}$,

U. Straumann ${ }^{41}$, D. Sunar ${ }^{4}$, T. Sykora ${ }^{4}$, V. Tchoulakov ${ }^{9}$, G. Thompson ${ }^{19}$, P.D. Thompson ${ }^{3}$, T. Toll ${ }^{11}$, F. Tomasz ${ }^{16}$,

T.H. Tran ${ }^{27}$, D. Traynor ${ }^{19}$, T.N. Trinh ${ }^{21}$, P. Truöl ${ }^{41}$, I. Tsakov ${ }^{34}$, B. Tseepeldorj ${ }^{35}$, G. Tsipolitis ${ }^{11, j}$, I. Tsurin ${ }^{39}$,

J. Turnau ${ }^{7}$, E. Tzamariudaki ${ }^{26}$, K. Urban ${ }^{15}$, D. Utkin ${ }^{24}$, A. Valkárová ${ }^{32}$, C. Vallée ${ }^{21}$, P. Van Mechelen ${ }^{4}$,

A. Vargas Trevino ${ }^{11}$, Y. Vazdik ${ }^{25}$, S. Vinokurova ${ }^{11}$, V. Volchinski ${ }^{38}$, G. Weber ${ }^{12}$, R. Weber ${ }^{40}$, D. Wegener ${ }^{8}$,

C. Werner ${ }^{14}$, M. Wessels ${ }^{11}$, C. Wissing ${ }^{11}$, R. Wolf ${ }^{14}$, E. Wünsch ${ }^{11}$, S. Xella ${ }^{41}$, V. Yeganov ${ }^{38}$, J. Žáček ${ }^{32}$, J. Zálešák ${ }^{31}$,

Z. Zhang ${ }^{27}$, A. Zhelezov ${ }^{24}$, A. Zhokin ${ }^{24}$, Y.C. Zhu ${ }^{11}$, T. Zimmermann ${ }^{40}$, H. Zohrabyan ${ }^{38}$, F. Zomer ${ }^{27}$

${ }^{1}$ I. Physikalisches Institut der RWTH, Aachen, Germany ${ }^{1}$

2 Vinca Institute of Nuclear Sciences, Belgrade, Serbia

3 School of Physics and Astronomy, University of Birmingham, Birmingham, $\mathrm{UK}^{\mathrm{m}}$

${ }^{4}$ Inter-University Institute for High Energies ULB-VUB, Brussels, Universiteit Antwerpen, Antwerpen, Belgium ${ }^{\mathrm{n}}$

5 National Institute for Physics and Nuclear Engineering (NIPNE), Bucharest, Romania

${ }^{6}$ Rutherford Appleton Laboratory, Chilton, Didcot, $\mathrm{UK}^{\mathrm{m}}$

7 Institute for Nuclear Physics, Cracow, Poland ${ }^{\circ}$

${ }^{8}$ Institut für Physik, Universität Dortmund, Dortmund, Germany ${ }^{1}$

9 Joint Institute for Nuclear Research, Dubna, Russia 
10 CEA, DSM/DAPNIA, CE-Saclay, Gif-sur-Yvette, France

11 DESY, Hamburg, Germany

12 Institut für Experimentalphysik, Universität Hamburg, Hamburg, Germany ${ }^{1}$

13 Max-Planck-Institut für Kernphysik, Heidelberg, Germany

14 Physikalisches Institut, Universität Heidelberg, Heidelberg, Germany ${ }^{1}$

15 Kirchhoff-Institut für Physik, Universität Heidelberg, Heidelberg, Germany ${ }^{1}$

16 Institute of Experimental Physics, Slovak Academy of Sciences, Košice, Slovak Republic ${ }^{\mathrm{p}}$

17 Department of Physics, University of Lancaster, Lancaster, UK ${ }^{\mathrm{m}}$

18 Department of Physics, University of Liverpool, Liverpool, $\mathrm{UK}^{\mathrm{m}}$

19 Queen Mary and Westfield College, London, UK ${ }^{\mathrm{m}}$

20 Physics Department, University of Lund, Lund, Sweden ${ }^{\mathrm{q}}$

21 CPPM, CNRS/IN2P3 - Univ. Mediterranee, Marseille, France

22 Departamento de Fisica Aplicada, CINVESTAV, Mérida, Yucatán, Méxicor ${ }^{\mathrm{r}}$

23 Departamento de Fisica, CINVESTAV, México ${ }^{r}$

24 Institute for Theoretical and Experimental Physics, Moscow, Russia

25 Lebedev Physical Institute, Moscow, Russia ${ }^{\mathrm{s}}$

26 Max-Planck-Institut für Physik, München, Germany

27 LAL, Univ Paris-Sud, CNRS/IN2P3, Orsay, France

${ }^{28}$ LLR, Ecole Polytechnique, IN2P3-CNRS, Palaiseau, France

${ }^{29}$ LPNHE, Universités Paris VI and VII, IN2P3-CNRS, Paris, France

30 Faculty of Science, University of Montenegro, Podgorica, Montenegro ${ }^{\mathbf{s}}$

31 Institute of Physics, Academy of Sciences of the Czech Republic, Praha, Czech Republic ${ }^{\mathrm{t}}$

32 Faculty of Mathematics and Physics, Charles University, Praha, Czech Republic ${ }^{\mathrm{t}}$

33 Dipartimento di Fisica Università di Roma Tre and INFN Roma 3, Roma, Italy

${ }^{34}$ Institute for Nuclear Research and Nuclear Energy, Sofia, Bulgarias

35 Institute of Physics and Technology of the Mongolian Academy of Sciences, Ulaanbaatar, Mongolia

36 Paul Scherrer Institut, Villigen, Switzerland

37 Fachbereich C, Universität Wuppertal, Wuppertal, Germany

38 Yerevan Physics Institute, Yerevan, Armenia

39 DESY, Zeuthen, Germany

40 Institut für Teilchenphysik, ETH, Zürich, Switzerland ${ }^{\mathrm{u}}$

41 Physik-Institut der Universität Zürich, Zürich, Switzerland ${ }^{u}$

Received: 28 November 2007 / Revised version: 18 December 2007 /

Published online: 19 February 2008 - (C) Springer-Verlag / Società Italiana di Fisica 2008

\begin{abstract}
The production of isolated photons in deep-inelastic scattering $e p \rightarrow e \gamma X$ is measured with the H1 detector at HERA. The measurement is performed in the kinematic range of negative four-momentum transfer squared $4<Q^{2}<150 \mathrm{GeV}^{2}$ and a mass of the hadronic system $W_{X}>50 \mathrm{GeV}$. The analysis is based on a total integrated luminosity of $227 \mathrm{pb}^{-1}$. The production cross section of isolated photons with a transverse energy in the range $3<E_{\mathrm{T}}^{\gamma}<10 \mathrm{GeV}$ and pseudorapidity range $-1.2<\eta^{\gamma}<1.8$ is measured as a function of $E_{\mathrm{T}}^{\gamma}, \eta^{\gamma}$ and $Q^{2}$. Isolated photon cross sections are also measured for events with no jets or at least one hadronic jet. The measurements are compared with predictions from Monte Carlo generators modelling the photon radiation from the quark and the electron lines, as well as with calculations at leading and next to leading order in the strong coupling. The predictions significantly underestimate the measured cross sections.
\end{abstract}

\footnotetext{
a Also at Faculty of Physics, University of Bucharest, Bucharest, Romania

b Also at Comenius University, Bratislava, Slovak Republic

c Also at Rechenzentrum, Universität Wuppertal, Wuppertal, Germany

d Also at CERN, Geneva, Switzerland

e e-mail: diaconu@cppm.in2p3.fr

f Also at Max-Planck-Institut für Physik, München, Germany

g Also at DESY and University Hamburg, Helmholtz Humboldt Research Award

h Supported by a scholarship of the World Laboratory Björn Wiik Research Project

i Also at University of P.J. Šafárik, Košice, Slovak Republic

j Also at Physics Department, National Technical University, Zografou Campus, 15773 Athens, Greece
}

$\mathrm{k}$ This project is co-funded by the European Social Fund $(75 \%)$ and National Resources (25\%) - (EPEAEK II) PYTHAGORAS II

${ }^{1}$ Supported by the Bundesministerium für Bildung und Forschung, FRG, under contract numbers 05 H1 1GUA /1, 05 H1 1PAA / 1, 05 H1 1PAB /9, 05 H1 1PEA /6, 05 H1 1VHA / 7 and $05 \mathrm{H} 11 \mathrm{VHB} / 5$

m Supported by the UK Particle Physics and Astronomy Research Council, and formerly by the UK Science and Engineering Research Council

n Supported by FNRS-FWO-Vlaanderen, IISN-IIKW and IWT and by Interuniversity Policy

- Partially Supported by Polish Ministry of Science and Higher Education, grant PBS/DESY/70/2006

p Supported by VEGA SR grant no. 2/7062/ 27 


\section{Introduction}

Isolated photons originating from the hard interaction in high energy collisions involving hadrons are a sensitive probe of perturbative quantum chromodynamics (QCD) $[1,2]$, as the photons are largely insensitive to the effects of hadronisation. A good understanding of the standard model (SM) production mechanism of isolated photons is also important for searches of new particles decaying to photons at hadron colliders.

The production of isolated photons ${ }^{1}$ has been studied at various experiments. Cross sections measured in fixed target $p N$ experiments (e.g. [3]) show a steeper decrease with photon transverse momentum, $P_{\mathrm{T}}^{\gamma}$, than predicted by nextto-leading order (NLO) QCD calculations. The CDF [4-7] and D0 $[8,9]$ experiments at the Tevatron have measured the isolated photon production cross section in $p \bar{p}$ collisions. Whereas D0 finds good agreement with a NLO QCD calculation, the CDF data show a somewhat steeper $P_{\mathrm{T}}^{\gamma}$ dependence than predicted. Measurements of the photon production in $e^{+} e^{-}$collisions have also been performed at LEP [10-13]. At HERA, prompt photon cross sections have been measured by the H1 and ZEUS experiments [14-16] in photoproduction, where the negative four-momentum transfer squared $Q^{2}$ of the exchanged virtual photon is close to zero, and showed reasonable agreement with NLO calculations. An analysis of the isolated photon cross section in deep-inelastic scattering (DIS) with $Q^{2}$ larger than $35 \mathrm{GeV}^{2}$ has been published by ZEUS [17].

The measurement of isolated photons in DIS provides a test of perturbative QCD in a kinematic range with two hard scales: the transverse energy of the emitted photon $E_{\mathrm{T}}^{\gamma}$ and $Q^{2}$. Isolated photons in DIS are produced at lowest order $\left(\alpha^{3} \alpha_{s}^{0}\right)$ as shown in Fig. 1. Already at this order a jet can be produced in the hadronic final state in addition to jets associated with the proton remnant, due to the electron or photon recoil.

The final state photon may be emitted by a quark $(Q Q)$ and by wide angle radiation from the lepton $(L L)$. The interference contribution $(L Q)$ is expected to be small. Since the photon and the scattered electron are well separated in the present analysis, low angle QED radiation is suppressed. The $Q Q$ contribution has two different origins: the direct radiation of a photon from the quark and the fragmentation of the quark into a jet containing a photon which carries a large fraction of the jet energy. This quarkto-photon fragmentation contribution is suppressed by the isolation requirement for the photon.

This paper presents a measurement of isolated photon production in DIS $e+p \rightarrow e+\gamma+X$. Photons are identified

\footnotetext{
q Supported by the Swedish Natural Science Research Council

r Supported by CONACYT, México, grant 48778-F

s Supported by the Deutsche Forschungsgemeinschaft

t Supported by the Ministry of Education of the Czech Republic under the projects LC527 and INGO-1P05LA259

u Supported by the Swiss National Science Foundation

1 Photons coupling to the interacting partons are often called "prompt" in contrast to photons from hadron decays or those emitted by leptons.
}
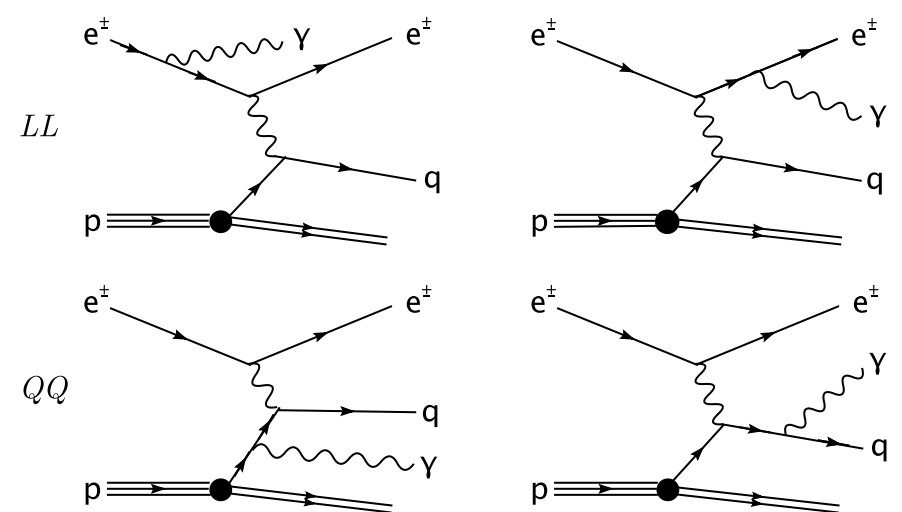

Fig. 1. Leading order diagrams for isolated photon production in DIS. The upper diagrams illustrate isolated photon production by radiation from the electron line $(L L)$, while the lower diagrams correspond to production via radiation from the quark $(Q Q$, without the contribution from quark fragmentation)

using a multivariate analysis of the shapes of the calorimeter energy deposits to reduce the background from neutral hadrons and their decay products. The photons are then used together with the other particles in the event, with the exception of the scattered electron, to reconstruct jets. The isolation of the photon is ensured by requiring that it carries at least $90 \%$ of the transverse momentum of the jet containing the photon. Isolated photons with transverse energy $3<E_{\mathrm{T}}^{\gamma}<10 \mathrm{GeV}$ and pseudorapidity $-1.2<$ $\eta^{\gamma}<1.8$ are selected in DIS events in the kinematic regime $4<Q^{2}<150 \mathrm{GeV}^{2}$, inelasticity $y>0.05$ and a mass of the hadronic system $W_{X}>50 \mathrm{GeV}$. The production of additional jets besides the photon jets in these events is also investigated. The current analysis significantly extends the kinematic range probed by the ZEUS measurement [17]. The results are compared to a recent leading order (LO), $\mathcal{O}\left(\alpha^{3} \alpha_{s}^{0}\right)$, calculation $[18,19]$ and to predictions of the Monte Carlo (MC) models PYTHIA [20], simulating the $Q Q$ process, and RAPGAP [21] for the $L L$ process. The cross sections for a photon plus at least one jet are further compared to a NLO calculation [22].

\section{$2 \mathrm{H} 1$ detector}

A detailed description of the $\mathrm{H} 1$ detector can be found in $[23,24]$. In the following, only detector components relevant to this analysis are briefly discussed. The origin of the $\mathrm{H} 1$ coordinate system is the nominal $e p$ interaction point, with the direction of the proton beam defining the positive $z$-axis (forward direction). Transverse momenta are measured in the $x-y$ plane. Polar $(\theta)$ and azimuthal $(\phi)$ angles are measured with respect to this reference system. The pseudorapidity is defined to be $\eta=-\ln \tan (\theta / 2)$.

In the central region $\left(20^{\circ}<\theta<165^{\circ}\right)$ the interaction region is surrounded by the central tracking system, which consists of a silicon vertex detector, drift chambers and multi-wire proportional chambers, all operated 
within a solenoidal magnetic field of $1.16 \mathrm{~T}$. The trajectories of charged particles are measured in the central tracker with a transverse momentum resolution of $\sigma\left(P_{\mathrm{T}}\right) / P_{\mathrm{T}} \simeq 0.005 P_{\mathrm{T}} / \mathrm{GeV} \oplus 0.015$. The forward tracking detector and the backward drift chamber (operated in 1999-2000) or backward proportional chamber (for 2003-2005) measure tracks of charged particles at smaller $\left(7^{\circ}<\theta<25^{\circ}\right)$ and larger $\left(155^{\circ}<\theta<175^{\circ}\right)$ polar angle than the central tracker, respectively. In each event the $e p$ interaction vertex is reconstructed from the measured charged tracks.

The liquid argon (LAr) sampling calorimeter [25] surrounds the tracking chambers. It has a polar angle coverage of $4^{\circ}<\theta<154^{\circ}$ and full azimuthal acceptance. It consists of an inner electromagnetic section with lead absorbers and an outer hadronic section with steel absorbers. The calorimeter is divided into eight wheels along the beam axis, each of them segmented in $\phi$ into eight modules, separated by small regions of inactive material. The electromagnetic and the hadronic sections are highly segmented in the transverse and the longitudinal direction with about 44000 cells in total. The granularity is larger in the electromagnetic part and increasing in both sections in the forward direction. For particles coming from the $e p$ interaction region, the laterally projected cell size in the electromagnetic part varies between $5 \times 5 \mathrm{~cm}^{2}$ in the forward and at most $7 \times 13 \mathrm{~cm}^{2}$ in the central region. The longitudinal segmentation in the different wheels varies from three (central) to four (forward) layers in the electromagnetic and from four to six in the hadronic section. The first electromagnetic layer has a thickness of about 3 to 6 radiation lengths for particles coming from the interaction region. Electromagnetic shower energies are measured with a precision of $\sigma(E) / E=12 \% / \sqrt{E / \mathrm{GeV}} \oplus 1 \%$ and hadronic energies with $\sigma(E) / E=50 \% / \sqrt{E / \mathrm{GeV}} \oplus$ $2 \%$, as determined in test beam experiments $[26,27]$. In the backward region $153^{\circ}<\theta<178^{\circ}$, particle energies are measured by a lead-scintillating fibre spaghetti calorimeter (SpaCal) [28].

The luminosity is determined from the rate of the Bethe-Heitler process $e p \rightarrow e p \gamma$, measured using a photon detector located close to the beam pipe at $z=-103 \mathrm{~m}$, in the backward direction.

DIS events at $Q^{2}$ values up to $150 \mathrm{GeV}^{2}$ are triggered by the energy deposition of the scattered electron in the SpaCal. For events with the scattered electron entering the $\mathrm{SpaCal}$ at low radii, additional trigger signals are required from the central drift chambers [29-31] and from the central proportional chambers [32-34]. The trigger efficiency for DIS events containing an electron in the Spacal angular acceptance with an energy above $10 \mathrm{GeV}$ is greater than $98 \%$.

\section{Monte Carlo simulations}

Monte Carlo simulations are used to correct the data for detector acceptances, inefficiencies and migrations and to compare the measured cross sections with MC model predictions.

The two generators PYTHIA [20] and RAPGAP [21] are used to generate events with photons produced in the hard interaction. PYTHIA simulates the contribution of photons radiated from the struck quark $(Q Q)$. The contribution of photons radiated by the electron $(L L)$ is simulated using RAPGAP and denoted "RAPGAP rad." in the following. The small contribution from interference [18] is neglected. Both generators calculate the hard partonic interaction in LO QCD $\mathcal{O}\left(\alpha^{3} \alpha_{s}^{0}\right)$. Higher order QCD radiation is modelled using initial and final state parton showers in the leading log approximation [35]. The fragmentation into hadrons is simulated using the LUND string model [36] as implemented in JETSET[37]. The simulations use the CTEQ6L proton parton densities [38].

The measurements presented in this paper show that the data is well described by the two MC contributions if PYTHIA is scaled by a factor 2.3 and RAPGAP is not scaled. This combined "scaled signal MC" is used to correct the data, whereas the unscaled MC prediction ("signal MC") is compared to the cross section measurements.

As an alternative, the HERWIG [39] generator is used to model the $Q Q$ contribution. HERWIG simulates the fragmentation into hadrons through the decay of colourless parton clusters and uses the equivalent-photon approximation for the incoming photon beam. Isolated photon production in DIS is derived approximately as Compton scattering between the photon and a quark. This approximation is not valid for $Q^{2}$ above a few $\mathrm{GeV}^{2}$, therefore HERWIG is only used to estimate the systematic uncertainties due to the fragmentation model.

The main SM background is due to photons produced in hadron decays in DIS events. It is modelled using the RAPGAP generator, with initial and final state radiation switched off. This contribution is denoted by "RAPGAP non-rad." in the following.

The multivariate shower shape analysis used to identify the photons requires high statistics samples of shower simulations in the whole phase space of energy and pseudorapidity. Samples of events containing single particles are simulated. In each sample, corresponding to single photons or single neutral hadrons decaying to photons, the particles are uniformly generated in pseudorapidity and energy. These samples are generically named "single particle samples" in the following.

All generated events are passed through a full GEANT [40] simulation of the $\mathrm{H} 1$ detector and through the same reconstruction and analysis programs as used for the data.

\section{Event selection}

The event sample used in this analysis was collected with the H1 detector at HERA in the period 1999 to 2005 at a centre-of-mass energy of $319 \mathrm{GeV}$. The corresponding integrated luminosity is $227 \mathrm{pb}^{-1}$. In a first step, DIS events 

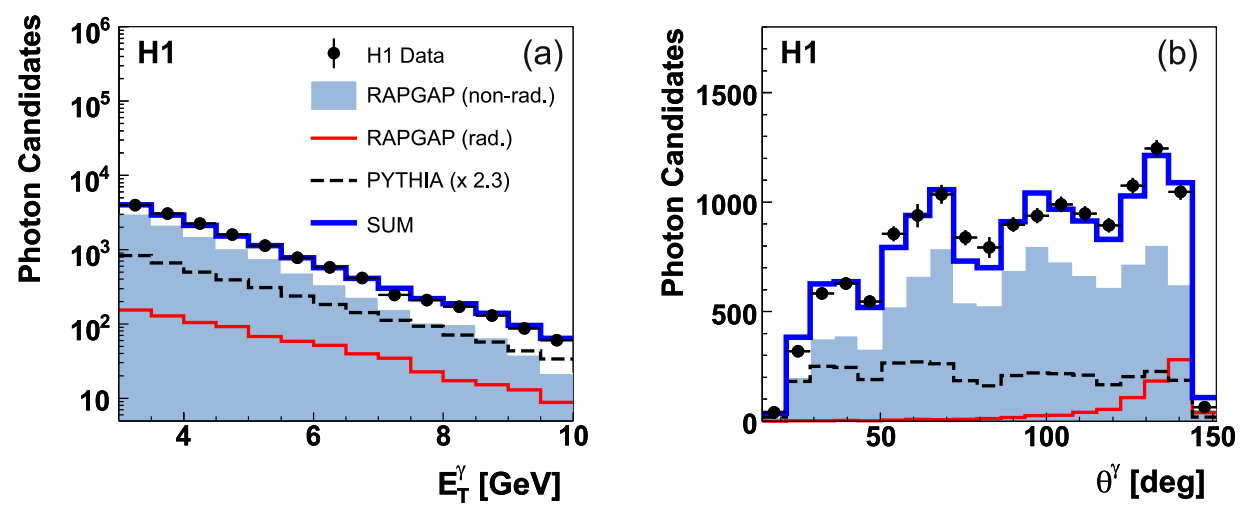

Fig. 2. Distributions of $\mathbf{a} E_{\mathrm{T}}^{\gamma}$ and $\mathbf{b} \theta^{\gamma}$ for isolated photon candidates in the final event sample. Data are shown as points with error bars. The bold solid histogram shows the sum of the expectation from RAPGAP (non-rad.) for neutral hadron background (shaded), from PYTHIA for radiation from the quark scaled by a factor of 2.3 (dashed line) and from RAPGAP (rad.) for radiation from the electron (solid line). The unshaded area corresponds to the estimated isolated photon contribution (RAPGAP(rad.) plus PYTHIA $\times 2.3)$

are selected with the scattered electron ${ }^{2}$ measured in the SpaCal. In a second step, a subsample of DIS events with an isolated photon candidate in the LAr calorimeter is selected.

\subsection{Selection of DIS events}

DIS events are selected with the scattered electron identified in the SpaCal as a compact electromagnetic cluster [41] with an energy $E_{e}>10 \mathrm{GeV}$ and a polar angle $\theta_{e}<177^{\circ}$. Matching signals in the backward tracking chambers are required for electron candidates with $E_{e}<$ $18 \mathrm{GeV}$. The scattering angle of the electron is determined from the measured impact position in the backward tracking chamber, the position of the energy cluster in the SpaCal and the reconstructed primary vertex.

Background from events at low $Q^{2}$, in which the electron escapes through the beam pipe and a hadron fakes the electron signature, is suppressed by the requirement that the difference $\Sigma\left(E-p_{z}\right)$ between the total energy and the longitudinal momentum be in the range $35<\Sigma\left(E-p_{z}\right)$ $<70 \mathrm{GeV}$, where the sum includes all measured hadronic final state particles and the scattered electron.

Non-ep background is removed by restricting the $z$-coordinate of the event vertex to be within $\pm 40 \mathrm{~cm}$ of the average vertex position and by requiring at least one good track in the central tracking system with the polar angle $30<\theta<150^{\circ}$ and not associated to the electron.

The energy $E_{e}$ and polar angle $\theta_{e}$ of the scattered electron candidate are used to reconstruct $y$ and $Q^{2}$ according to $Q^{2}=2 E_{e}^{0} E_{e}\left(1+\cos \theta_{e}\right)$ and $y=1-E_{e}\left(1-\cos \theta_{e}\right)$ $/\left(2 E_{e}^{0}\right)$, where $E_{e}^{0}$ is the electron beam energy. The events are selected in the kinematic region $4<Q^{2}<150 \mathrm{GeV}^{2}$ and $y>0.05$.

\footnotetext{
2 The analysis uses data from periods when the beam lepton was either a positron or an electron. Unless otherwise stated, the term electron refers to both the electron and the positron.
}

\subsection{Selection of isolated photon candidates and jets}

Photon candidates are identified as clusters in the electromagnetic section of the LAr calorimeter with a transverse energy $3<E_{\mathrm{T}}^{\gamma}<10 \mathrm{GeV}$ and pseudorapidity $-1.2<\eta^{\gamma}$ $<1.8$ in the $\mathrm{H} 1$ laboratory frame. The candidates are rejected if they are close to inactive regions between calorimeter modules [25] or if a track geometrically matches the electromagnetic cluster with a distance of closest approach to the cluster's barycentre of less than $20 \mathrm{~cm}$. Neutral hadrons that decay into multiple photons constitute the main background. In most cases such decay photons are merged into one electromagnetic cluster, which tends to have a wider transverse distribution than that of a single photon. The transverse radius ${ }^{3} R_{\mathrm{T}}$ of the photon candidate cluster is therefore required to be smaller than $6 \mathrm{~cm}$. In addition, the invariant mass of the cluster, when combined with the closest neighbouring electromagnetic cluster with an energy above $80 \mathrm{MeV}$, must be larger than $300 \mathrm{MeV}$. This requirement rejects candidates that originate from $\pi^{0}$ decays with two photons reconstructed in separate clusters. Only events with exactly one photon candidate are accepted. Less than $1 \%$ of the events are rejected because more than one photon candidate is found.

The mass of the final state hadronic system is reconstructed from the four-momenta of the incoming electron $\left(p_{e}\right)$ and proton $\left(p_{p}\right)$, the scattered electron $\left(p_{e}^{\prime}\right)$ and the photon candidate $\left(p_{\gamma}\right)$ as $W_{X}=\sqrt{\left(p_{e}+p_{p}-p_{e}^{\prime}-p_{\gamma}\right)^{2}}$. The contribution from elastic Compton scattering $(p+e \rightarrow p+$ $e+\gamma)$ is suppressed by requiring $W_{X}>50 \mathrm{GeV}$.

Final state hadrons are reconstructed from deposits in the LAr calorimeter in combination with tracking information. Following the so-called "democratic" procedure $[42,43]$, the photon candidate and the reconstructed hadrons in each event are combined into massless jets using the $k_{\mathrm{T}}$ algorithm [44]. The algorithm is used with a $P_{\mathrm{T}^{-}}$ weighted recombination scheme and with the separation parameter $R_{0}$ set to 1 . Jets are selected with a transverse

\footnotetext{
3 For a definition of the transverse radius see Sect. 5 .
} 

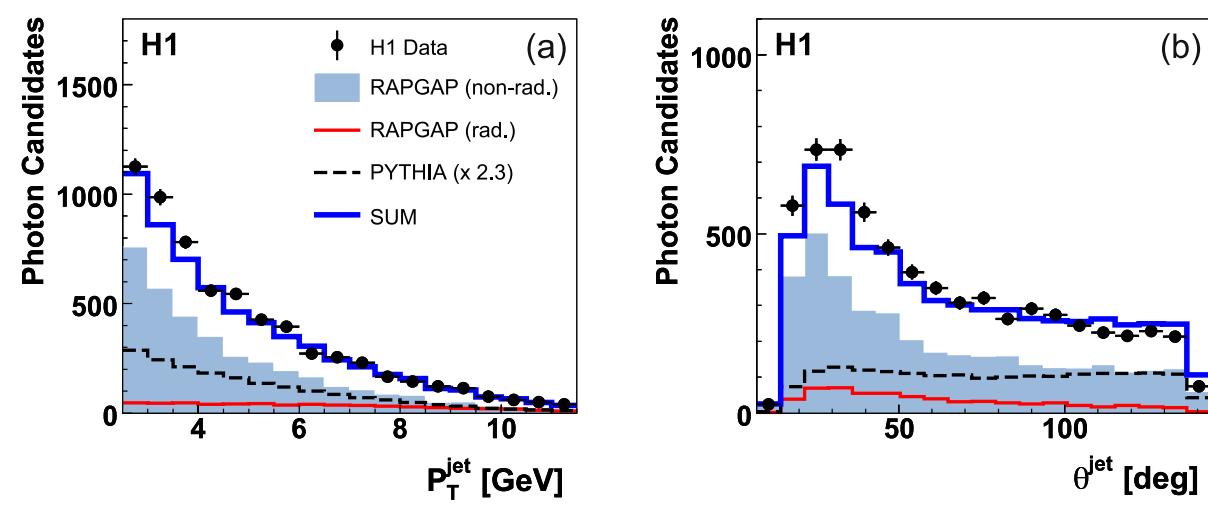

Fig. 3. Distributions of a the transverse momentum and $\mathbf{b}$ the polar angle of the hadronic jet with the highest transverse momentum in events with an isolated photon candidate. The data are compared to the MC predictions, as described in the caption of Fig. 2 momentum of $P_{\mathrm{T}}^{\text {jet }}>2.5 \mathrm{GeV}$ and a pseudorapidity in the range $-2.0<\eta^{\text {jet }}<2.1$. Due to the harder kinematical cuts for the photon candidate there is always a jet containing the photon candidate, called the photon-jet. All other jets are classified as hadronic jets. For hadronic jets the $\eta^{\text {jet }}$ range is restricted to $-1.0<\eta^{\text {jet }}<2.1$. According to the $\mathrm{MC}$ simulation, the hadronic jets are well correlated to jets reconstructed by the same jet algorithm on parton level (partonic jets) even at low transverse energies. To ensure isolation of the photon, the fraction $z$ of the transverse energy of the photon-jet carried by the photon candidate has to be larger than $90 \%$. This definition of the isolation requirement is stable against infrared divergences and thus well suited for comparisons with perturbative QCD calculations. The isolation requirement largely suppresses background from photons produced in hadron decays.

The distributions of the transverse energy and of the polar angle of the isolated photon candidates are shown in Fig. 2 together with the MC predictions for the scaled signal and the background. The sum of the MCs describes the data well.

The samples of events with either no hadronic jet or at least one hadronic jet are called "photon plus no-jets" and "photon plus jet", respectively. The $P_{\mathrm{T}}^{\text {jet }}$ and $\theta^{\text {jet }}$ distributions for the hadronic jet with the largest transverse momentum are shown in Fig. 3. The sum of the scaled signal and background MCs describe reasonably well the data, with a slight deficit for jets at low polar angles.

A total of 14670 events with a scattered electron and an isolated photon candidate are selected, of which 6495 have at least one additional hadronic jet.

\section{Photon signal extraction}

\subsection{Shower shape analysis}

The extraction of the photon signal exploits the fine granularity of the electromagnetic part of the LAr calorimeter. In order to discriminate between signal photons and the background from neutral hadrons and their decay products, the calorimeter cluster corresponding to the isolated photon candidate is further analysed using the following six shower shape variables calculated from the measurements of the individual cells composing the cluster [45]:
1. The fraction of the energy of the electromagnetic cluster contained in the cell with the largest energy deposit ("hottest cell").

2. The fraction of the energy of the electromagnetic cluster contained in four or eight (depending on the granularity of the calorimeter) contiguous cells in the first two calorimeter layers. The cells include the hottest cell and are chosen to maximise the energy which they contain ("hot core"). This and the first variable are sensitive to the compactness of the cluster in the calorimeter. The values of these variables are on average larger for photons than for the background.

3. The fraction of the cluster's energy detected in the first calorimeter layer ("layer 1"), which is expected to be larger on average for multi-photon clusters than for those initiated by a single photon.

4. The transverse ${ }^{4}$ symmetry $S_{\mathrm{T}}$ of a cluster defined as the ratio of the spread (defined by root mean squared) of the transverse cell distributions along the two principal axes. A photon cluster is expected to be symmetric with $S_{\mathrm{T}}$ values close to unity, whereas multi-photon clusters are typically more asymmetric and yield lower $S_{\mathrm{T}}$ values.

5. The transverse radius of the cluster defined as the square root of the second central transverse moment $R_{\mathrm{T}}=\sqrt{\mu_{2}}$, where the $k$ 'th central transverse moment of the cells distribution is given by $\mu_{k}=\left\langle|\mathbf{r}-\langle\mathbf{r}\rangle|^{k}\right\rangle$. Here $\mathbf{r}$ is the transverse projection of a cell position and $\langle\mathbf{r}\rangle=\left(\sum_{i=0}^{n} E_{i} \mathbf{r}_{i}\right) / \sum E_{i}$ the energy weighted average of the cell positions $\mathbf{r}_{i}$ in the plane transverse to the photon direction. As explained in Sect. 4.2, only events with a cluster candidate of small transverse radius $R_{\mathrm{T}}<6 \mathrm{~cm}$ are selected for the multivariate analysis.

6. The transverse kurtosis $K_{\mathrm{T}}$ is defined as $K_{\mathrm{T}}=\mu_{4} /$ $\left(\mu_{2}\right)^{2}-3$. It specifies how strongly the energy distribution is peaked and is equal to zero for a Gaussian distribution.

The discrimination power of signal and background becomes weaker at high transverse energies, where the multiphoton clusters become more similar to a single photon

\footnotetext{
4 In the context of the cluster shape analysis the transverse plane is defined as perpendicular to the direction of the photon candidate.
} 

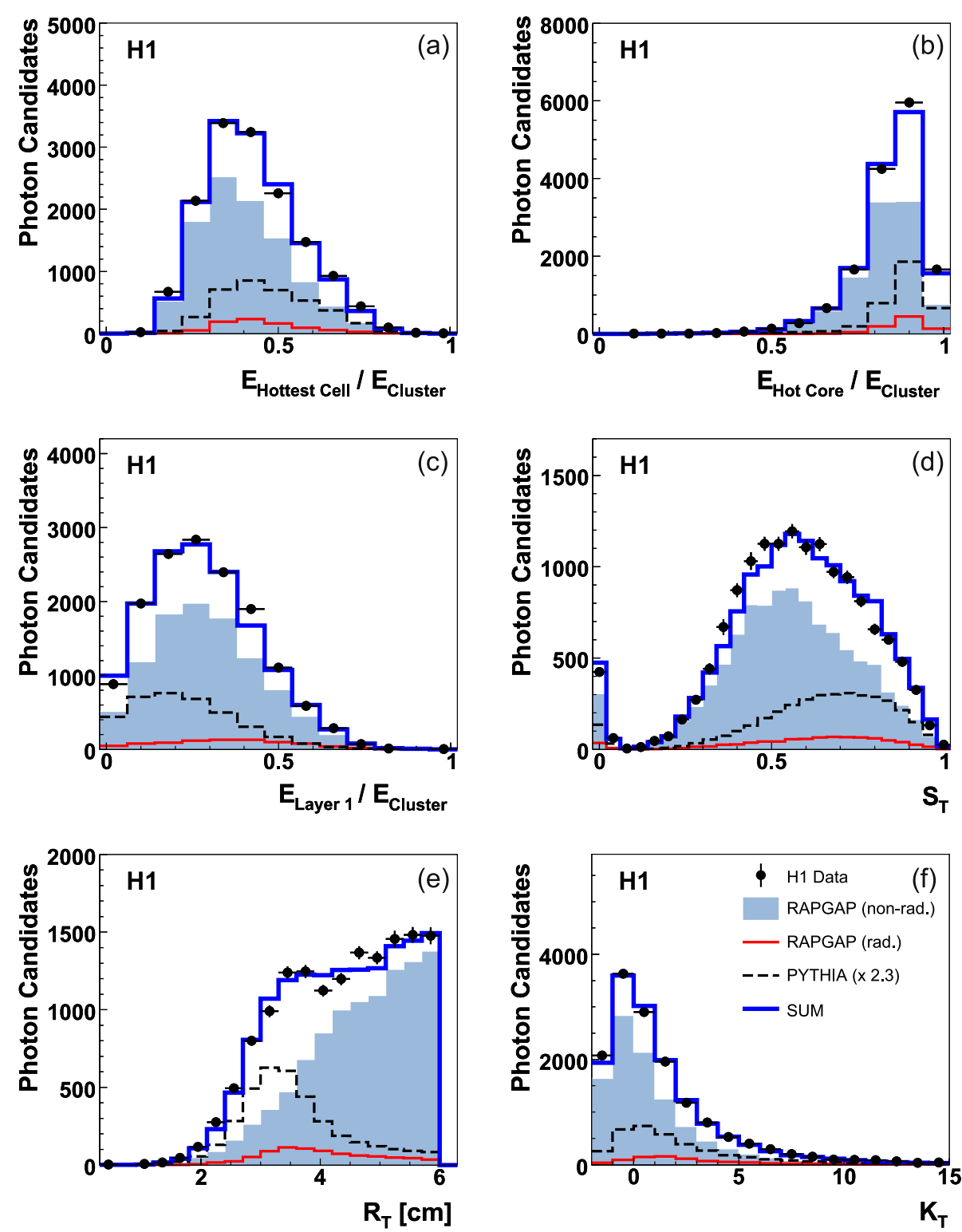

Fig. 4. Distributions of the six variables that are used to define the discriminant for isolated photon identification: a hottest cell fraction, $\mathbf{b}$ fraction of the hot core, c first layer fraction, d transverse symmetry $S_{\mathrm{T}}$, e transverse radius $R_{\mathrm{T}}$ and $\mathbf{f}$ transverse kurtosis $K_{\mathrm{T}}$. The data are shown with the $\mathrm{MC}$ predictions described in the caption to Fig. 2. The shape difference between RAPGAP (rad.) and PYTHIA arises from the different distributions in phase space (see Fig. 2b) cluster. Therefore events with $E_{\mathrm{T}}^{\gamma}>10 \mathrm{GeV}$ are excluded from the measurement, as described in Sect. 4.2.

The distributions of the six shower shape variables are shown for the isolated photon candidates in Fig. 4. The data are compared with the sum of the background and the scaled signal MC distributions. A good agreement is observed.

\subsection{Signal extraction}

In order to discriminate between single photons (signal) and single neutral hadrons (background), probability density functions $p_{\gamma, \mathrm{bg}}^{i=1,6}$ are determined for the six shower shape variables, using simulated "single particles events", described in Sect. 3. The signal probability density functions $p_{\gamma}^{i}$ are simulated using single photon events whereas the background probability density functions $p_{\mathrm{bg}}^{i}$ are approximated using a sample of events containing single neu- tral hadrons $\left(\pi^{0}, \eta, \eta^{\prime}, \rho, \omega, K^{\star}, K_{L}^{0}, K_{S}^{0}, n\right.$ and $\left.\bar{n}\right)$. The relative contributions of the various neutral hadrons species are taken as predicted by the RAPGAP generator. In particular, $\pi^{0}$ and $\eta$ mesons contribute to $90 \%$ of the background.

An overlap of clusters of different particles can occur due to large multiplicities specific to the hadronic environment in DIS. The overlap affects the photon candidate cluster shape. It is found to be important only for the background and leads to a loss of photon candidates due to the distortion of the transverse cluster radius, which then exceeds in most cases the upper limit of $6 \mathrm{~cm}$ required in the analysis (cf. Sect. 4.2). This loss due to cluster overlap is modelled by supressing the background single particles according to a probability $p_{\text {co }}$ proportional to the transverse area of the cluster $p_{\text {co }}=a R_{\mathrm{T}}^{2}$ with $a=0.004 \mathrm{~cm}^{-2}$. The constant $a$ is determined by a comparison of single particles event samples with full MC simulation in phase space regions where sufficient statistics are available. 
The multi-dimensional photon and background probability densities are taken as the product of the respective shower shape densities $P_{\gamma, \mathrm{bg}}=\prod_{i=1,6} p_{\gamma, \mathrm{bg}}^{i}$. For each event a discriminator $(D)$ is formed, which is defined as the photon probability density divided by the sum of the probability densities for photons and background $D=P_{\gamma} /$ $\left(P_{\gamma}+P_{\mathrm{bg}}\right)$. The discriminator has in general larger values for isolated photons than for the decay photons. Figure 5 shows the discriminator distribution for the data together with the predictions of the background and the scaled signal MC. The data are well described by the sum of the MC predictions.

Since the shower shape densities vary significantly as a function of the cluster energy and depend on the granularity of the LAr calorimeter, the discriminator is determined in bins of $\left(E_{\mathrm{T}}^{\gamma}, \eta^{\gamma}\right)$, with three intervals in $E_{\mathrm{T}}^{\gamma}$ and five in $\eta^{\gamma}$. The intervals in $\eta^{\gamma}$ correspond to the different wheels of the calorimeter. The contributions of photons and neutral hadrons in any of the 15 analysis bins is determined by independent minimum- $\chi^{2}$ fits to the data discriminator distributions. In each $\left(E_{\mathrm{T}}^{\gamma}, \eta^{\gamma}\right)$ bin, the $\chi^{2}$ function is defined to be

$$
\chi^{2}=\sum_{i} \frac{\left(N_{\mathrm{data}, i}-N_{\mathrm{bg}} d_{\mathrm{bg}, i}-N_{\mathrm{sig}} d_{\mathrm{sig}, i}\right)^{2}}{\sigma_{\mathrm{data}, i}^{2}+N_{\mathrm{bg}}^{2} \sigma_{\mathrm{bg}, i}^{2}+N_{\mathrm{sig}}^{2} \sigma_{\mathrm{sig}, i}^{2}},
$$

where the sum runs over the bins of the discriminator distributions $(D) . N_{\text {data }, i}$ is the number of data events in the $i$ 'th bin. $d_{\mathrm{sig}, i}$ and $d_{\mathrm{bg}, i}$ denote the $i$ 'th bin content of the signal and background discriminator distribution, respectively, normalised to unity. The $\sigma_{i}$ represent the associated statistical errors. $N_{\text {sig }}$ and $N_{\mathrm{bg}}$, represent the number of signal and background events respectively, and are determined by the fit. If the content in any data histogram bin is small $\left(N_{\text {data }, i}<7\right)$, adjacent bins are merged. The fit is performed separately for the three samples selected as presented in Sect. 4.2: inclusive, photon plus no-jets and photon plus jet. The fit quality is acceptable in all differential bins.

The total number of isolated photons is obtained by summing the $N_{\text {sig }}$ from all analysis bins. As a result $4372 \pm$

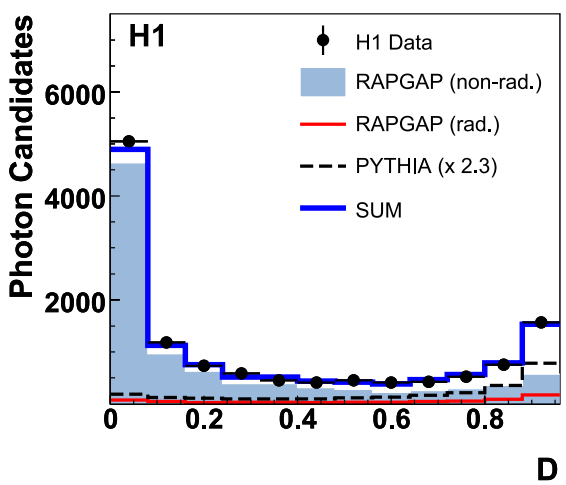

Fig. 5. The distribution of the discriminator $(D)$ used in the identification of isolated photon candidates for events that have passed the event selection. The data are compared to the $\mathrm{MC}$ predictions described in the caption of Fig. 2
145 signal events are attributed to the inclusive data sample. In the photon plus no-jets and photon plus jet subsamples $1755 \pm 106$ and $2606 \pm 95$ signal events are found, respectively. The signal fraction is $29 \%$ in the inclusive sample, $20 \%$ for the photon plus no-jets sample and $39 \%$ for the photon plus jet sample.

\subsection{Cross section determination}

In each bin of the kinematic variables, the cross section $\sigma$ is computed from the number of events with photons in the corresponding bin as $\sigma=N_{\text {sig }} /(\mathcal{L} \epsilon)$, where $\mathcal{L}$ is the luminosity. The correction factor $\epsilon$ takes into account the acceptance, trigger and reconstruction efficiencies, and migration between the bins. It is calculated using the scaled signal MC. Bin averaged cross sections are quoted in all tables and figures. The total inclusive cross section is obtained by summing the measured cross sections from all 15 analysis bins. The bin averaged single differential cross sections $\mathrm{d} \sigma / \mathrm{d} E_{\mathrm{T}}^{\gamma}$ and $\mathrm{d} \sigma / \mathrm{d} \eta^{\gamma}$ are obtained accordingly by summing all corresponding bins in $\eta^{\gamma}$ and $E_{\mathrm{T}}^{\gamma}$, respectively. The single differential cross section $\mathrm{d} \sigma / \mathrm{d} Q^{2}$ is determined by fitting the discriminator distributions separately in five different bins in $Q^{2}$. In these fits, the signal and background discriminator distributions in each $E_{\mathrm{T}}^{\gamma}$ and $\eta^{\gamma}$ bin are assumed to be independent of $Q^{2}$. It has been verified that the variation of the $Q^{2}$ dependence has indeed a negligible effect.

\section{Systematic uncertainties}

Two additional event samples are used for the determination of systematic errors and in-situ energy calibration. The first sample, containing Bethe-Heitler events, $e p \rightarrow e \gamma p$, consists of events with an electron reconstructed in the LAr calorimeter, a photon in the SpaCal and nothing else in the detector. The second, complementary, sample is selected by requiring an electron in the SpaCal and a photon in the LAr calorimeter and no other particle detected in the event. Such events originate to a large part from deeply virtual Compton scattering $e p \rightarrow e \gamma p$. These independent event selections, denoted BH and DVCS respectively, provide a clean sample of electromagnetic clusters in the LAr calorimeter.

The effects of the different systematic errors on the cross section are evaluated by applying variations to the MC simulation. The following uncertainties are considered:

- The measured shower shape variables in the BH and DVCS event samples are compared to simulated single particle photons and electrons, respectively. The uncertainty on the shower shape simulation is estimated by distorting the discriminating variables within the limits deduced from the differences observed for the control samples (BH and DVCS) between data and simulation. The fits for the signal extraction (Sect. 5.2) are repeated with the distorted distributions of the discriminating variables. The resulting systematic error on the 
total inclusive cross section is $+10.2 \%$ and $-12.8 \%$. It varies between $11 \%$ and $25 \%$ for the single differential cross sections. The error increases with increasing $E_{\mathrm{T}}^{\gamma}$ and towards large $\eta^{\gamma}$ and is independent of $Q^{2}$.

- The uncertainty on the photon energy measurement is estimated using the BH and DVCS control samples. For the $\mathrm{BH}$ events the cluster transverse energy is compared with the track measurement. For DVCS events the energy of the cluster is compared to the energy calculated using the double angle method [46,47]. The photon energy scale uncertainty estimated with this method varies from $1 \%$ for photons detected in the backward region to $4 \%$ for forward photons. The resulting error on the total inclusive cross section is $+3.6 \%$ and $-2.6 \%$.

- An uncertainty of $3 \mathrm{mrad}\left(4 \mathrm{mrad}\right.$ for $\eta_{\gamma}>1.4$ ) is attributed to the measurement of the polar angle of the photon. This uncertainty is determined by comparing the polar angle measurements of the track and the cluster for identified electrons in the $\mathrm{BH}$ control sample. The resulting error on the total inclusive cross section is $+0.1 \%$ and $-0.7 \%$.

- An uncertainty of up to $2 \%$ is attributed to the energy of the scattered electron and an uncertainty of $2 \mathrm{mrad}$ to the measurement of the scattering angle. The resulting error on the total inclusive cross section is $+1.9 \%$ and $-2.9 \%$.

- A $3 \%$ uncertainty is attributed to the energy of hadronic final state objects [48]. This affects the total cross section by $+1.2 \%$ and $-0.7 \%$.

- A $5 \%$ uncertainty is applied for the correction of the cluster overlaps in the signal extraction procedure, corresponding to half the size of the correction.

- The uncertainty attributed to the model dependence of the acceptance corrections is derived from the differences between the acceptances determined with the PYTHIA and HERWIG simulations. A resulting error of $5 \%$ is found for the inclusive and the photon plus jet and $10 \%$ for the photon plus no-jets cross sections.

- The uncertainty of the ratio of the $L L$ and $Q Q$ contributions for the acceptance corrections is taken into account by varying the scaling factor for PYTHIA from 1.5 to 3 . The resulting error on the total inclusive cross section is $+1.4 \%$ and $-0.8 \%$. For the double differential cross section, a systematic error of up to $5 \%$ is found. In regions with $\eta^{\gamma}>-0.6$, the systematic error is below $1 \%$.

- An uncertainty of $1 \%$ is attributed to the simulation of the trigger efficiency.

- The uncertainty on the track reconstruction efficiency results in an error of $\pm 0.3 \%$ for the total inclusive cross section.

- The uncertainty on the conversion probability of the photons before entering the calorimeter results in a systematic error of $2 \%$ of the cross sections.

- The uncertainty on the luminosity measurement is $3.4 \%$.

In each analysis bin the individual effects of these experimental uncertainties are combined in quadrature. The systematic uncertainty obtained on the total inclusive cross section is $+13.6 \%$ and $-15.5 \%$. The largest contribution to this uncertainty arises from the systematic effects attributed to the description of the shower shapes, which is partially correlated among measurement bins.

\section{Theoretical predictions}

The measured cross sections are compared to the unscaled MC prediction as discussed in Sect. 3. In addition, data are compared with fixed order QCD calculations, described in this section. A $\mathrm{LO} \mathcal{O}\left(\alpha^{3} \alpha_{s}^{0}\right)$ calculation $[18,19]$ is used. The photon plus jet cross sections are further compared to a NLO $\mathcal{O}\left(\alpha^{3} \alpha_{s}\right)$ QCD calculation [22] which is only available for the photon plus jets phase space selection. In the calculations, the cross section $\sigma(e p \rightarrow e \gamma X)$ is obtained by convoluting the parton-level cross sections (for instance $\hat{\sigma}(e q \rightarrow e \gamma q)$ at LO) with the proton parton density functions.

The $Q Q$ contribution is dominated by the direct radiation of the photon from the quark involved in the parton level process, but also contains the contribution from quark fragmentation to a photon $[49,50]$. The direct part can be calculated in perturbation theory. The fragmentation contribution is described by a DIS matrix element $e q \rightarrow e q$ convoluted with a process independent quark-to-photon fragmentation function determined from data and denoted by $D_{q \rightarrow \gamma}(z)$. Here $z$ is the fraction of the quark's momentum carried by the photon. Already at leading order, the parton-level cross section contains an infrared divergence due to the emission of a photon collinear to the quark. It is factorised into the fragmentation function at a factorisation scale $\mu_{\mathrm{F}}$. This singularity at LO makes a NLO prediction for the inclusive cross section considerably involved.

So far, only two measurements exist that give direct information on the quark-to-photon fragmentation function. These measurements were made by the LEP experiments ALEPH [43] and OPAL [51]. Only the ALEPH measurement has the precision to allow a determination of $D_{q \rightarrow \gamma}(z)$. The LO calculation used in this analysis is based on the ALEPH LO parametrisation of $D_{q \rightarrow \gamma}(z)[42,43]$. The NLO calculation uses the fragmentation function from [52]. In the MC models the contribution from fragmentation is not included.

For photons plus no-jets the quark-to-photon fragmentation enters already at lowest order $\left(\alpha^{3} \alpha_{s}^{0}\right)$ [19], in contrast to the photon plus jet sample where the fragmentation contribution is of the order $\alpha^{3} \alpha_{s}^{1}$. Since the contribution from fragmentation is suppressed by the requirement of the photon being isolated, the present analysis has no sensitivity for a determination of $D_{q \rightarrow \gamma}(z)$.

In the $L L$ subprocess, the photon is radiated by the lepton. In the present analysis, the reconstruction of the photon and the electron in different parts of the detector ensures that the two particles are separated, hence the $L L$ term contains no collinear singularities. The interference term $(L Q)$, which differs in sign for $e^{+} p$ and $e^{-} p$ scattering, is included in the calculations. It contributes less than $3 \%$ to the cross section [18]. 
The calculations of the isolated photon cross section are made for the same ratio of luminosities for $e^{+} p(47.9 \%)$ and $e^{-} p(52.1 \%)$ scattering as for the data. The cuts in the theoretical calculations are adjusted to correspond to the experimental cuts described in this paper. The calculations use the same jet algorithm, applied on parton level, as used for the data analysis. The CTEQ6L [38] leading order parametrisation of proton parton distributions is used. Different proton parton density functions are found to change the predictions by $5 \%-10 \%$. The LO and NLO predictions are compared to the data after a correction for hadronisation effects. The correction factors $f_{\text {had }}$ are defined as the ratio of the cross sections calculated from hadrons to those from partons and are determined from the scaled signal MC. The same jet algorithm as for the data is applied on parton and on hadron level. The uncertainty of the correction factor is estimated by comparing the correction factors obtained from PYTHIA with those from HERWIG. The correction for the total inclusive cross section is $-14 \%$ with an associated uncertainty of $5 \%$. For the differential cross sections the corrections are at most $-30 \%$. The correction is largest for low photon energies and in forward direction of the photon because of the degraded resolution of the isolation parameter $z$. The combined uncertainties of the theoretical predictions from hadronisation corrections and proton parton distributions amount to up to $11 \%$.

The NLO calculation of the photon plus jet cross sections includes processes with an additional gluon, either as the incoming parton or in the final state, as well as virtual corrections. The renormalisation and factorisation scales are set to $\mu_{\mathrm{R}}=\mu_{\mathrm{F}}=\sqrt{Q^{2}+\left(P_{\mathrm{T}}^{\text {jet }}\right)^{2}}$. Theoretical uncertainties are estimated by varying $\mu_{\mathrm{R}}$ and $\mu_{\mathrm{F}}$ independently by a factor two up and down. These uncertainties are below $3 \%$ and lower than the uncertainties from the choice of the proton parton distributions and from the hadronisation corrections.

\section{Results}

The isolated photon cross sections presented below are given for the phase space defined in Table 1. Bin averaged differential cross sections are presented in Figs. 6-10 and in Tables 2-5. For all measurements the total uncertainty is dominated by systematics. The results are compared with the signal MC predictions (unscaled PYTHIA plus RAPGAP) and with the LO and NLO calculations. The factors $f_{\text {had }}$ for the correction of the theoretical calculations from parton to hadron level are given in the cross section tables.

\subsection{Inclusive isolated photon cross sections}

The measured inclusive isolated photon cross section is

$$
\sigma(e p \rightarrow e \gamma X)=50.3 \pm 1.7 \text { (stat) }{ }_{-7.8}^{+6.8} \text { (syst) pb . }
$$

The LO calculation predicts a cross section of $28.6 \mathrm{pb}$, while the signal MC expectation is $26.4 \mathrm{pb}$. Thus both pre-
Table 1. Phase space region in which isolated prompt photon cross sections are measured together with the definition of jets. Kinematics are defined in the H1 laboratory frame

\begin{tabular}{l}
\hline Isolated photon cross section phase space \\
$3<E_{\mathrm{T}}^{\gamma}<10 \mathrm{GeV}$ \\
$-1.2<\eta^{\gamma}<1.8$ \\
$z=E_{\mathrm{T}}^{\gamma} / E_{\mathrm{T}}^{\text {photon-jet }}>0.9$ \\
$E_{e}>10 \mathrm{GeV}$ \\
$153<\theta_{e}<177^{\circ}$ \\
$4<Q^{2}<150 \mathrm{GeV}^{2}$ \\
$W_{X}>50 \mathrm{GeV}$ \\
$y>0.05$ \\
Inclusive \\
$\sqrt{s}=319 \mathrm{GeV}$ \\
$k_{\mathrm{T}}$ algorithm with $P_{\mathrm{T}}$-weighted \\
recombination scheme $[44], R_{0}=1$ \\
$P_{\mathrm{T}}^{\text {jet }}>2.5 \mathrm{GeV}$ \\
$-1.0<\eta^{\text {jet }}<2.1$ (hadronic jet) \\
$-2.0<\eta^{\text {photon-jet }}<2.1$ (photon-jet) \\
Jet definition
\end{tabular}

Table 2. Differential cross sections for inclusive isolated photon production in the kinematic range specified in Table $1 . f_{\text {had }}$ denotes the hadronisation correction factor applied to the LO calculation

\begin{tabular}{|c|c|c|c|c|c|}
\hline \multicolumn{6}{|c|}{ H1 inclusive isolated photon cross sections } \\
\hline \multicolumn{2}{|c|}{$\begin{array}{c}E_{\mathrm{T}}^{\gamma} \\
{[\mathrm{GeV}]}\end{array}$} & $\mathrm{d} \sigma / \mathrm{d} E_{\mathrm{T}}^{\gamma}$ & $\begin{array}{c}\text { stat. } \\
{[\mathrm{pb} / \mathrm{GeV}]}\end{array}$ & syst. & $f_{\text {had }}$ \\
\hline 3.0 & 4.0 & 16.98 & \pm 1.20 & $\begin{array}{l}+2.79 \\
-2.61\end{array}$ & 0.78 \\
\hline 4.0 & 6.0 & 10.51 & \pm 0.47 & $\begin{array}{l}+1.50 \\
-1.86\end{array}$ & 0.89 \\
\hline 6.0 & 10.0 & 3.08 & \pm 0.20 & $\begin{array}{l}+0.46 \\
-0.60\end{array}$ & 0.98 \\
\hline \multicolumn{2}{|c|}{$\eta^{\gamma}$} & $\mathrm{d} \sigma / \mathrm{d} \eta^{\gamma}$ & $\begin{array}{l}\text { stat. } \\
{[\mathrm{pb}]}\end{array}$ & syst. & $f_{\text {had }}$ \\
\hline-1.2 & -0.6 & 26.15 & \pm 1.67 & $\begin{array}{l}+3.60 \\
-4.16\end{array}$ & 0.92 \\
\hline-0.6 & 0.2 & 20.69 & \pm 1.34 & $\begin{array}{l}+3.53 \\
-3.73\end{array}$ & 0.85 \\
\hline 0.2 & 0.9 & 15.83 & \pm 0.93 & $\begin{array}{l}+1.97 \\
-3.25\end{array}$ & 0.81 \\
\hline 0.9 & 1.4 & 9.57 & \pm 0.87 & $\begin{array}{l}+1.99 \\
-2.00\end{array}$ & 0.80 \\
\hline 1.4 & 1.8 & 5.50 & \pm 1.15 & $\begin{array}{l}+1.04 \\
-1.75\end{array}$ & 0.80 \\
\hline \multicolumn{2}{|c|}{$\begin{array}{c}Q^{2} \\
{\left[\mathrm{GeV}^{2}\right]}\end{array}$} & $\mathrm{d} \sigma / d Q^{2}$ & $\begin{array}{c}\text { stat. } \\
{\left[\mathrm{pb} / \mathrm{GeV}^{2}\right]}\end{array}$ & syst. & $f_{\text {had }}$ \\
\hline 4.0 & 10.0 & 2.48 & \pm 0.21 & $\begin{array}{l}+0.34 \\
-0.41\end{array}$ & 0.87 \\
\hline 10.0 & 20.0 & 1.17 & \pm 0.07 & $\begin{array}{l}+0.19 \\
-0.21\end{array}$ & 0.83 \\
\hline 20.0 & 40.0 & 0.52 & \pm 0.03 & $\begin{array}{l}+0.07 \\
-0.10\end{array}$ & 0.81 \\
\hline 40.0 & 80.0 & 0.235 & \pm 0.013 & $\begin{array}{l}+0.033 \\
-0.048\end{array}$ & 0.83 \\
\hline 80.0 & 150.0 & 0.063 & \pm 0.006 & $\begin{array}{l}+0.009 \\
-0.012\end{array}$ & 0.87 \\
\hline
\end{tabular}


$\mathrm{H} 1$ Isolated Photon Production in DIS
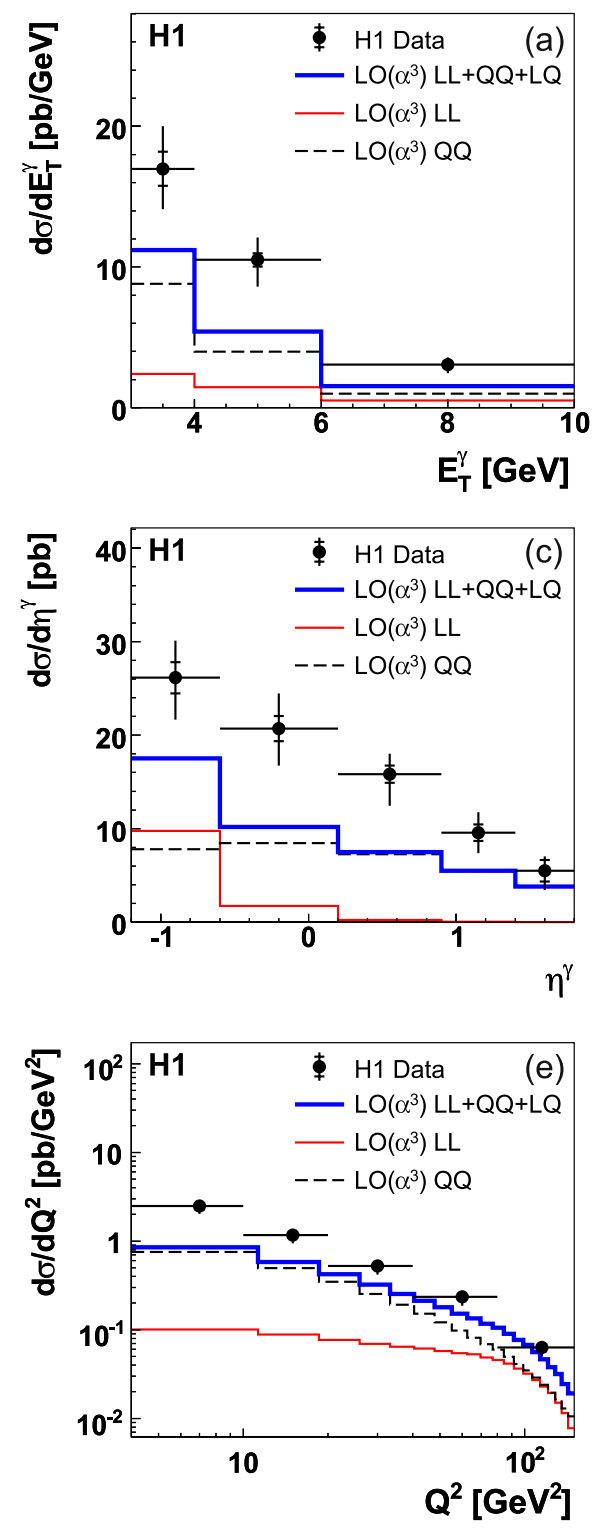
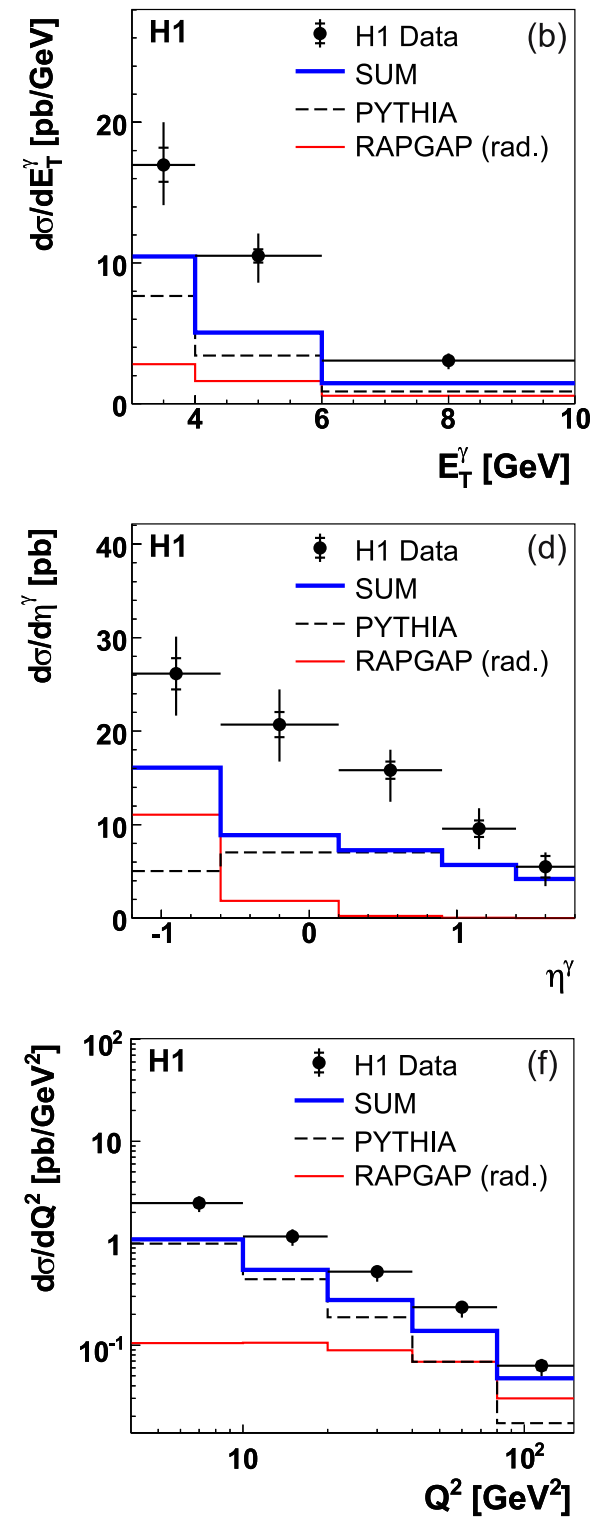

Fig. 6. Differential cross sections for inclusive isolated photon production $\mathrm{d} \sigma / \mathrm{d} E_{\mathrm{T}}^{\gamma}, \mathrm{d} \sigma / \mathrm{d} \eta^{\gamma}$, and $\mathrm{d} \sigma / \mathrm{d} Q^{2}$ in the kinematic range specified in Table 1. The inner error bars on the data points indicate the statistical error, the full error bars contain in addition the systematical errors added in quadrature. The cross sections in a, c, e are shown together with a leading order, $\mathcal{O}\left(\alpha^{3} \alpha_{s}^{0}\right)$, calculation corrected for hadronisation effects, $L L$ corresponding to radiation from the electron, $Q Q$ to radiation from the quark and $L Q$ to their interference. The same cross sections are shown in $\mathbf{b}, \mathbf{d}, \mathbf{f}$ together with the prediction from PYTHIA for photon emission from the quark and from RAPGAP (rad.) for emission from the electron dictions significantly underestimate the measured total inclusive cross section by almost a factor of two.

Differential cross sections $\mathrm{d} \sigma / \mathrm{d} E_{\mathrm{T}}^{\gamma}, \mathrm{d} \sigma / \mathrm{d} \eta^{\gamma}$ and $\mathrm{d} \sigma / \mathrm{d} Q^{2}$ are presented in Table 2 and in Fig. 6 .

The data are compared in the left panels of Fig. 6 with the LO predictions, displaying separately the $L L$ and $Q Q$ contributions. The LO calculation provides a reasonable description of the shapes of the data distributions in $E_{\mathrm{T}}^{\gamma}$ and $\eta^{\gamma}$, while the global normalisation is about a factor of two too low. The calculation is closest to the data at larger $Q^{2}$ and for backward photons $\left(\eta^{\gamma}<-0.6\right)$, where the $L L$ contribution is of similar magnitude to that of $Q Q$. For forward and central photons $\left(\eta^{\gamma}>-0.6\right)$, the $Q Q$ contribution dominates.

The measurements are compared in the right panels of Fig. 6 with the signal MC predictions. The estimations of the $Q Q$ processes by PYTHIA and of the $L L$ contribu- tions by RAPGAP agree well with the predictions from the $\mathrm{LO}$ calculation. Thus the conclusions for the comparison of the signal MC with the data are similar as for the LO calculation. This agreement between LO and the signal MC holds for all cross sections studied in this analysis and in the following the data are only compared with the LO calculation.

Figure 7 and Table 3 present the differential cross section as a function of $E_{\mathrm{T}}^{\gamma}$ in five different bins of $\eta^{\gamma}$, corresponding to the wheel structure of the LAr calorimeter. The shapes of all cross section distributions are reasonably well described by the LO calculation. A good description of the data can be obtained by a global scaling of the $Q Q$ contribution by a factor 2.3 and leaving the $L L$ normalisation unchanged. This is an indication that the observed excess in the data is mainly due to an underestimation of the $Q Q$ component. 
$\mathrm{H} 1$ Isolated Photon Production in DIS
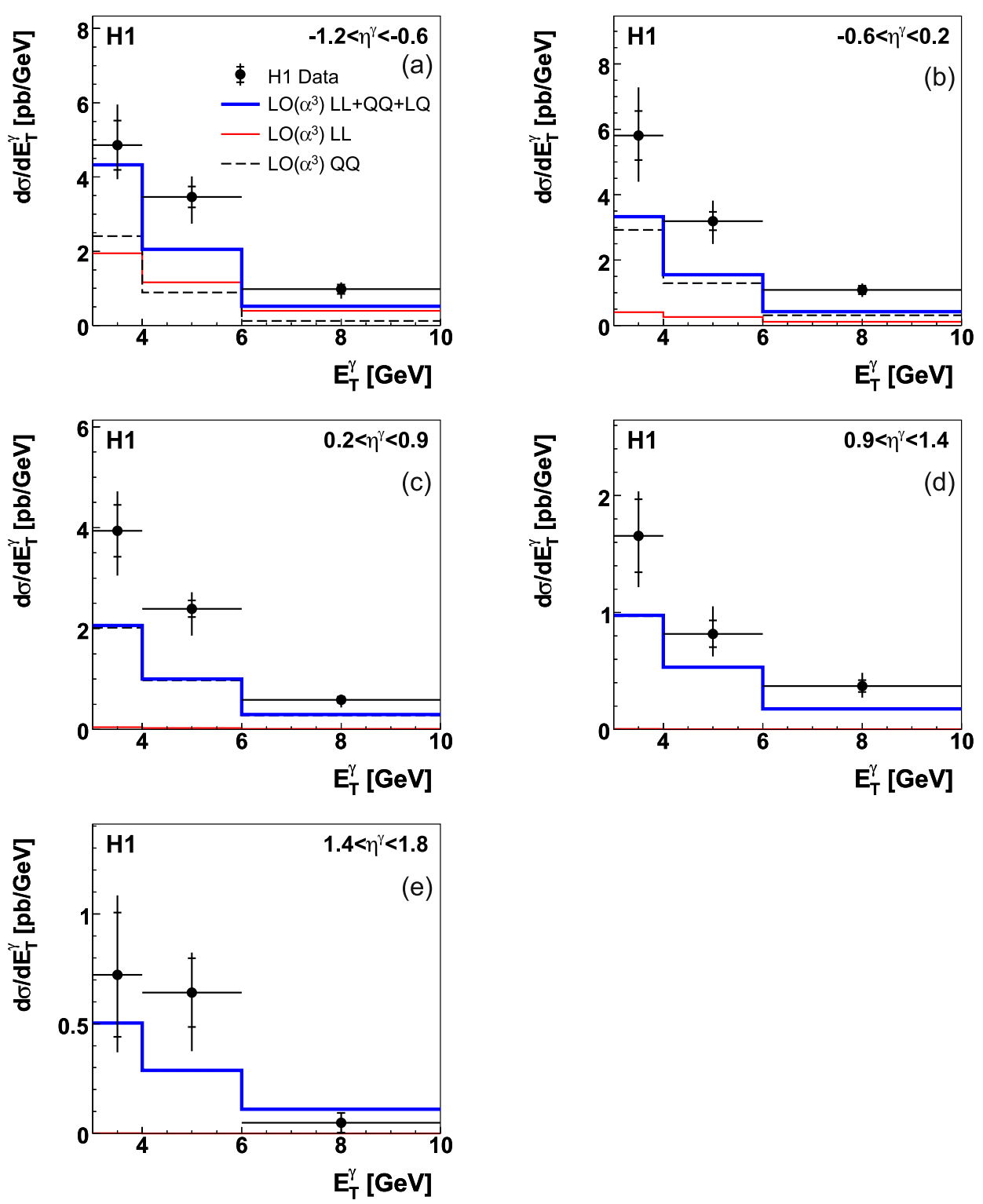

Fig. 7. Differential cross sections $\mathrm{d} \sigma / \mathrm{d} E_{\mathrm{T}}^{\gamma}$ for inclusive isolated photon production in the kinematic range specified in Table 1 , in $\eta^{\gamma}$ bins corresponding to the wheel structure of the LAr calorimeter (see text). The measurements are compared to a leading order $\mathcal{O}\left(\alpha^{3} \alpha_{s}^{0}\right)$ calculation (more details in the caption of Fig. 6). The $L L$ contribution is negligible for $\eta^{\gamma}>0.2$

At $Q^{2}>40 \mathrm{GeV}^{2}$, the agreement of the predictions with the data is somewhat better, as can be seen in Fig. 8 and Table 4 . The total inclusive cross section for $Q^{2}>40 \mathrm{GeV}^{2}$ is

$$
\sigma(e p \rightarrow e \gamma X)=14.0 \pm 0.8 \text { (stat) }{ }_{-2.1}^{+2.2} \text { (syst) pb } .
$$

The LO prediction of $10.3 \mathrm{pb}$ is about $30 \%$ below the data. The shapes of the $\mathrm{d} \sigma / \mathrm{d} E_{\mathrm{T}}^{\gamma}$ and $\mathrm{d} \sigma / \mathrm{d} \eta^{\gamma}$ distributions are well reproduced. The relative contribution of $L L$ is predicted to be higher than at low $Q^{2}$.

The present measurement is extrapolated to the phase space of the analysis performed by the ZEUS collaboration [17] $\left(Q^{2}>35 \mathrm{GeV}^{2}, y>0, E_{e}>10 \mathrm{GeV}, 139.8<\theta_{e}\right.$ $<171.9^{\circ}$ and $\left.5<E_{\mathrm{T}}^{\gamma}<10 \mathrm{GeV}\right)$. Due to the different angular coverage of the calorimeters of the two detectors, the extrapolation is not possible for $\eta^{\gamma}<-0.6$ because the photon-electron separation cannot be properly taken into account. Figure 9 shows the comparison of the H1 and ZEUS mesurements of the differential cross section $\mathrm{d} \sigma / \mathrm{d} \eta^{\gamma}$. A good agreement is observed. The LO calculation is also shown and is here corrected for hadronisation effects in contrast to the comparison in [18]. In the ZEUS analysis the photon radiation from the electron is neglected in the acceptance corrections and no $W_{X}$ cut is applied. A rough estimate shows that with the $W_{X}$ cut used by $\mathrm{H} 1$ and the acceptance calculation with the combination of PYTHIA and RAPGAP, the ZEUS cross section values are expected to be lowered by about $10 \%-30 \%$. A different photon isolation criterion is used in the ZEUS analysis which is not corrected for. Studies of isolated photons in photoproduction indicate that the two different isolation criteria give very similar results. The measurement presented in this pa- 
$\mathrm{H} 1$ Isolated Photon Production in DIS at $\mathrm{Q}^{2}>40 \mathrm{GeV}^{2}$

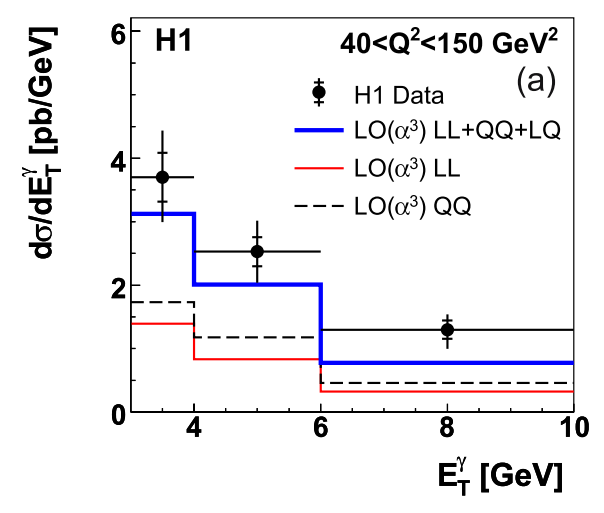

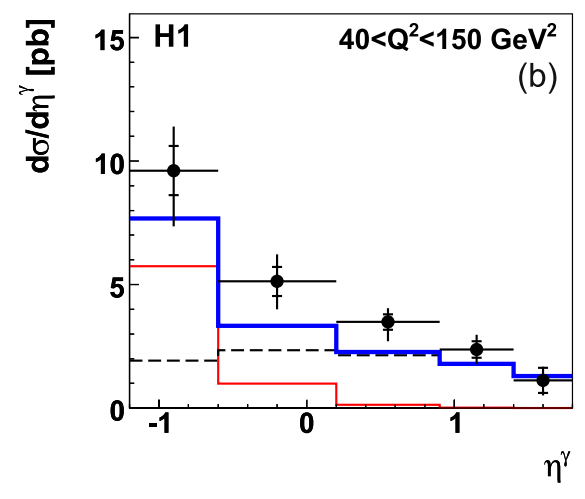

Fig. 8. Differential inclusive cross sections $\mathrm{d} \sigma / \mathrm{d} E_{\mathrm{T}}^{\gamma}(\mathbf{a})$ and $\mathrm{d} \sigma / \mathrm{d} \eta^{\gamma}$ (b) for isolated photon production in the kinematic range specified in Table 1 and the additional criterion $40<Q^{2}<150 \mathrm{GeV}^{2}$. The cross sections are compared to the leading order, $\mathcal{O}\left(\alpha^{3} \alpha_{s}^{0}\right)$, calculation (more details in the caption of Fig. 6)
Table 3. Differential cross sections for inclusive isolated photon production $\mathrm{d} \sigma / \mathrm{d} E_{\mathrm{T}}^{\gamma}$ in different $\eta^{\gamma}$ bins corresponding to the wheel structure of the LAr calorimeter (see text). The kinematic range is defined in Table 1. $f_{\text {had }}$ denotes the hadronisation correction factor applied to the LO calculation

\begin{tabular}{|c|c|c|c|c|c|}
\hline \multicolumn{6}{|c|}{ H1 inclusive isolated photon cross sections } \\
\hline & $\begin{array}{c}E_{\mathrm{T}}^{\gamma} \\
{[\mathrm{GeV}]}\end{array}$ & $\mathrm{d} \sigma / \mathrm{d} E_{\mathrm{T}}^{\gamma}$ & $\begin{array}{c}\text { stat. } \\
{[\mathrm{pb} / \mathrm{GeV}]}\end{array}$ & syst. & $f_{\text {had }}$ \\
\hline \multicolumn{6}{|c|}{$-1.2<\eta^{\gamma}<-0.6$} \\
\hline 3.0 & 4.0 & 4.86 & \pm 0.67 & $\begin{array}{l}+0.88 \\
-0.63\end{array}$ & 0.86 \\
\hline 4.0 & 6.0 & 3.46 & \pm 0.28 & $\begin{array}{l}+0.48 \\
-0.66\end{array}$ & 0.96 \\
\hline 6.0 & 10.0 & 0.98 & \pm 0.12 & $\begin{array}{l}+0.13 \\
-0.23\end{array}$ & 1.00 \\
\hline \multicolumn{6}{|c|}{$-0.6<\eta^{\gamma}<0.2$} \\
\hline 3.0 & 4.0 & 5.81 & \pm 0.75 & $\begin{array}{l}+1.27 \\
-1.20\end{array}$ & 0.76 \\
\hline 4.0 & 6.0 & 3.20 & \pm 0.28 & $\begin{array}{l}+0.56 \\
-0.65\end{array}$ & 0.88 \\
\hline 6.0 & 10.0 & 1.09 & \pm 0.13 & $\begin{array}{l}+0.15 \\
-0.17\end{array}$ & 0.99 \\
\hline \multicolumn{6}{|c|}{$0.2<\eta^{\gamma}<0.9$} \\
\hline 3.0 & 4.0 & 3.94 & \pm 0.51 & $\begin{array}{l}+0.59 \\
-0.72\end{array}$ & 0.72 \\
\hline 4.0 & 6.0 & 2.39 & \pm 0.16 & $\begin{array}{l}+0.28 \\
-0.51\end{array}$ & 0.84 \\
\hline 6.0 & 10.0 & 0.59 & \pm 0.06 & $\begin{array}{l}+0.09 \\
-0.14\end{array}$ & 0.96 \\
\hline \multicolumn{6}{|c|}{$0.9<\eta^{\gamma}<1.4$} \\
\hline 3.0 & 4.0 & 1.66 & \pm 0.31 & $\begin{array}{l}+0.22 \\
-0.31\end{array}$ & 0.69 \\
\hline 4.0 & 6.0 & 0.82 & \pm 0.12 & $\begin{array}{l}+0.21 \\
-0.16\end{array}$ & 0.82 \\
\hline 6.0 & 10.0 & 0.37 & \pm 0.05 & $\begin{array}{l}+0.10 \\
-0.09\end{array}$ & 0.96 \\
\hline \multicolumn{6}{|c|}{$1.4<\eta^{\gamma}<1.8$} \\
\hline 3.0 & 4.0 & 0.72 & \pm 0.28 & $\begin{array}{l}+0.23 \\
-0.21\end{array}$ & 0.70 \\
\hline 4.0 & 6.0 & 0.64 & \pm 0.16 & $\begin{array}{l}+0.09 \\
-0.22\end{array}$ & 0.81 \\
\hline 6.0 & 10.0 & 0.049 & \pm 0.045 & $\begin{array}{l}+0.009 \\
-0.016 \\
\end{array}$ & 0.94 \\
\hline
\end{tabular}

per significantly extends the kinematical region probed by ZEUS in photon transverse energy and pseudorapidity, and in $Q^{2}$.
Table 4. Differential cross sections for inclusive isolated photon production in the kinematic range specified in Table 1 and $40<Q^{2}<150 \mathrm{GeV}^{2}$. $f_{\text {had }}$ denotes the hadronisation correction factor applied to the LO calculation

\begin{tabular}{|c|c|c|c|c|c|}
\hline \multicolumn{6}{|c|}{$\begin{array}{l}\text { H1 inclusive isolated photon cross sections } \\
\text { for } Q^{2}>40 \mathrm{GeV}^{2}\end{array}$} \\
\hline & & $\mathrm{d} \sigma / \mathrm{d} E_{\mathrm{T}}^{\gamma}$ & $\begin{array}{l}\text { stat. } \\
{[\mathrm{pb} / \mathrm{GeV}]}\end{array}$ & syst. & $f_{\text {had }}$ \\
\hline 3.0 & 4.0 & 3.70 & \pm 0.39 & $\begin{array}{l}+0.63 \\
-0.59\end{array}$ & 0.80 \\
\hline 4.0 & 6.0 & 2.53 & \pm 0.23 & $\begin{array}{l}+0.43 \\
-0.43\end{array}$ & 0.87 \\
\hline 6.0 & 10.0 & 1.30 & \pm 0.15 & $\begin{array}{l}+0.19 \\
-0.27\end{array}$ & 0.96 \\
\hline & & $\mathrm{d} \sigma / \mathrm{d} \eta^{\gamma}$ & $\begin{array}{l}\text { stat. } \\
{[\mathrm{pb}]}\end{array}$ & syst. & $f_{\text {had }}$ \\
\hline-1.2 & -0.6 & 9.61 & \pm 1.00 & $\begin{array}{l}+1.48 \\
-2.02\end{array}$ & 0.97 \\
\hline-0.6 & 0.2 & 5.13 & \pm 0.59 & $\begin{array}{l}+0.92 \\
-0.97\end{array}$ & 0.86 \\
\hline 0.2 & 0.9 & 3.49 & \pm 0.32 & $\begin{array}{l}+0.45 \\
-0.72\end{array}$ & 0.78 \\
\hline 0.9 & 1.4 & 2.37 & \pm 0.33 & $\begin{array}{l}+0.49 \\
-0.47\end{array}$ & 0.76 \\
\hline 1.4 & 1.8 & 1.12 & \pm 0.51 & $\begin{array}{l}+0.21 \\
-0.34 \\
\end{array}$ & 0.73 \\
\hline
\end{tabular}

\subsection{Photon plus no-jets and photon plus jet cross sections}

The cross section for jet production in events with isolated photons is studied. The measurement is performed in the phase space defined for the inclusive cross section with an additional jet requirement or veto as shown in Table 1.

The measured total cross section for photon plus no-jets is

$$
\sigma(e p \rightarrow e \gamma Y)=18.8 \pm 1.2(\text { stat })_{-3.4}^{+3.3} \text { (syst) pb }
$$

where $Y$ contains no identified hadronic jet. The prediction of the LO calculation is $11.7 \mathrm{pb}$. The measured total cross section for the photon plus at least one jet is

$$
\sigma(e p \rightarrow e \gamma \text { jet } X)=31.6 \pm 1.2 \text { (stat) }{ }_{-4.8}^{+4.2} \text { (syst) } \mathrm{pb} .
$$

The prediction of the LO calculation is $16.7 \mathrm{pb}$. For both samples the predictions are significantly lower than 


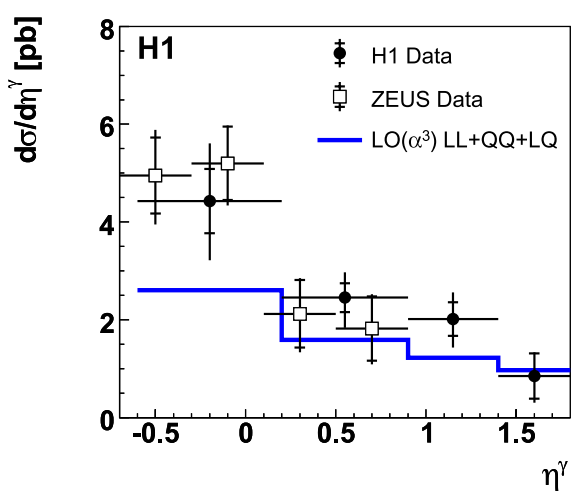

Fig. 9. Differential cross sections $\mathrm{d} \sigma / \mathrm{d} \eta^{\gamma}$ for the inclusive isolated photon production in comparison to the previous measurement by ZEUS [17] for $Q^{2}>35 \mathrm{GeV}^{2}, E_{e}^{\prime}>10 \mathrm{GeV}, 139.8<$ $\theta_{e}<171.9^{\circ}$ and $5<E_{\mathrm{T}}^{\gamma}<10 \mathrm{GeV}$. The additional condition $W_{X}>50 \mathrm{GeV}$ is used in the $\mathrm{H} 1$ analysis only (see Sect. 8 ). The cross sections are compared to the leading order, $\mathcal{O}\left(\alpha^{3} \alpha_{s}^{0}\right)$, calculation. In contrast to the comparison in [18], the calculation is here corrected for hadronisation effects

the data. The observed ratios of data to LO prediction are very similar to the inclusive isolated photon measurement. As for the inclusive sample, similar conclusions are found for the MC predictions.
A comparison to a NLO calculation is possible for the photon plus jet cross sections. The NLO calculation predicts a cross section of $20.2 \pm 0.6 \mathrm{pb}$, about $20 \%$ higher than the LO prediction but still roughly $35 \%$ lower than the data. The analysis performed using a higher cut on $P_{\mathrm{T}}^{\text {jet }}>4 \mathrm{GeV}$ lead to a similar disagreement between the data and the calculation.

The measured differential cross sections for the photon plus no-jets and photon plus jet selections are presented in Table 5. They are compared with the LO predictions in Fig. 10. For both samples the LO prediction describes the shapes of the $\mathrm{d} \sigma / \mathrm{d} E_{\mathrm{T}}^{\gamma}$ and $\mathrm{d} \sigma / \mathrm{d} \eta^{\gamma}$ distributions reasonably well and is furthest below the data at low $Q^{2}$, where the $Q Q$ term dominates. All four diagrams in Fig. 1 contribute to the photon plus no-jets and photon plus jet samples, but the $L L$ contribution is largely suppressed for the photon plus no-jets sample due to the cut on $W_{X}$. Since, at leading order $\mathcal{O}\left(\alpha_{s}^{0}\right)$, the quark fragmentation contribution to the cross section enters only the photon plus no-jets sample, the observed excess can not solely be attributed to an underestimation of that contribution. The cross section for photon plus jet production is roughly two times higher than for photon plus no-jets. This is in contrast to the inclusive $e p \rightarrow e X$ cross section, where topologies with an additional jet are suppressed by $\mathcal{O}\left(\alpha_{s}\right)$. The similar cross sections for photon events with or without additional jets

Table 5. Differential cross sections for the production of isolated photons accompanied by no or at least one hadronic jet in the kinematic range specified in Table 1. $f_{\text {had }}$ denotes the hadronisation correction factor applied to the LO and the NLO calculation

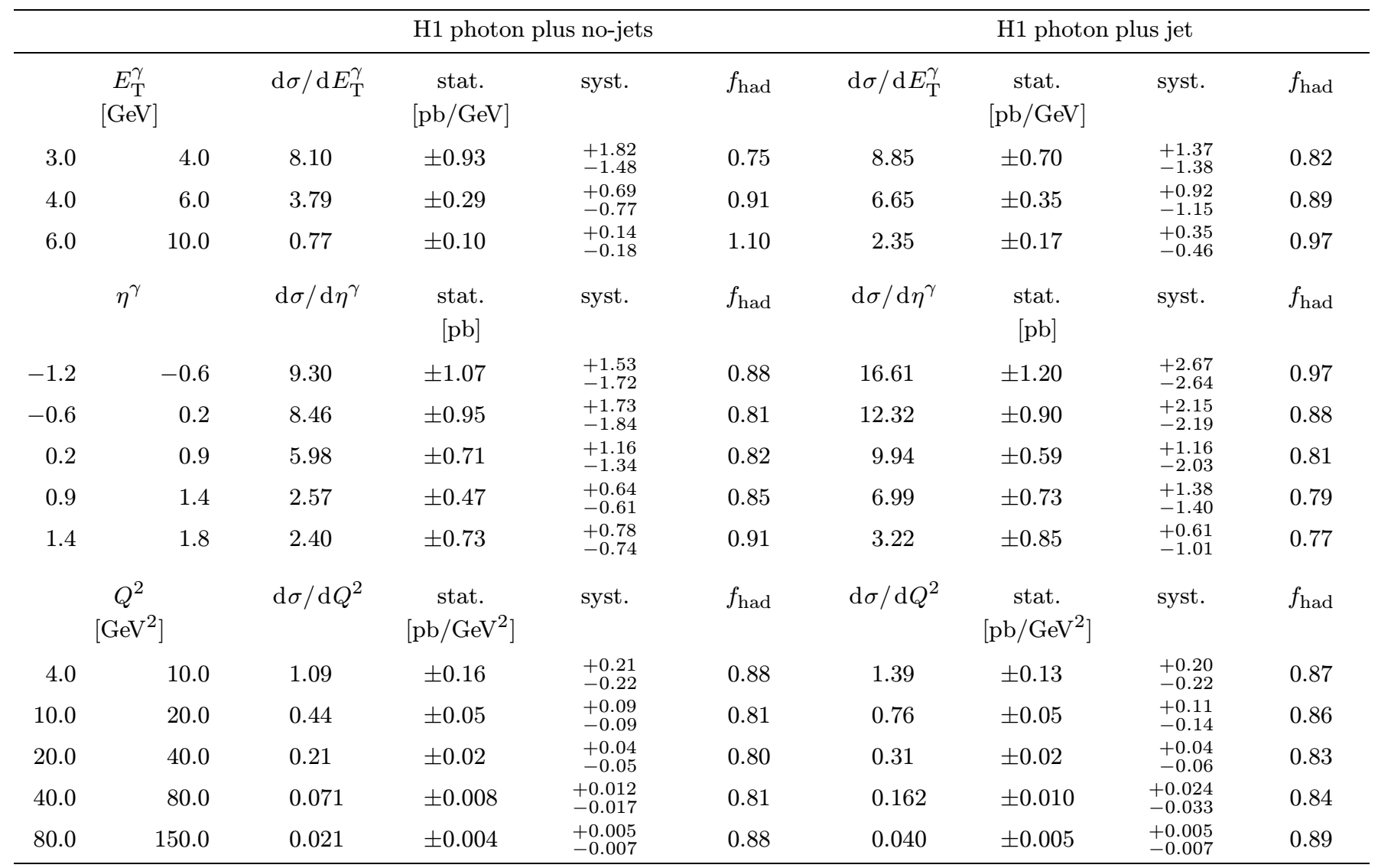



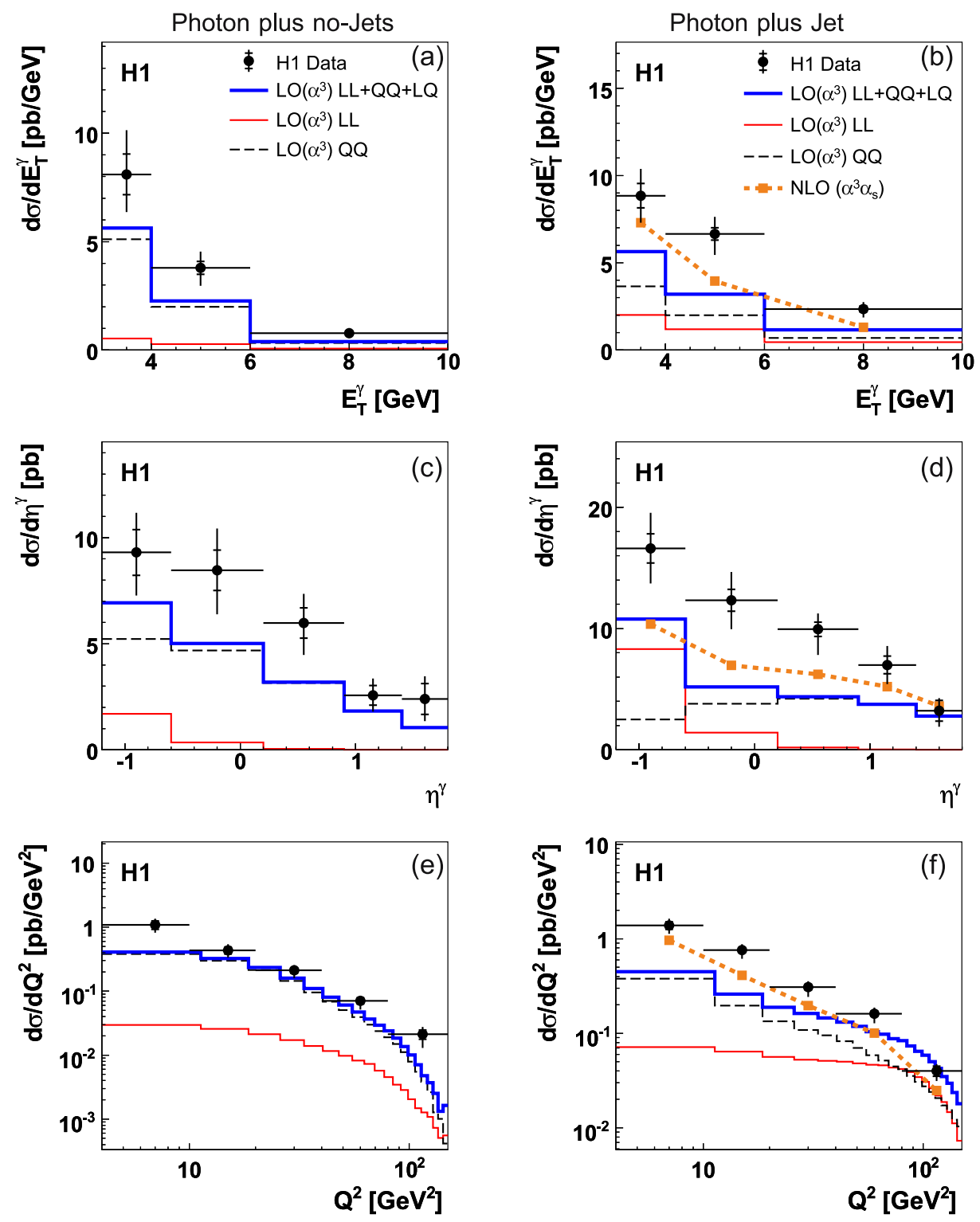

Fig. 10. Differential cross sections $\mathrm{d} \sigma / \mathrm{d} E_{\mathrm{T}}^{\gamma}, \mathrm{d} \sigma / \mathrm{d} \eta^{\gamma}$ and $\mathrm{d} \sigma / \mathrm{d} Q^{2}$ for photon plus no-jets $(\mathbf{a}, \mathbf{c}, \mathbf{e})$, and photon plus jet $(\mathbf{b}, \mathbf{d}, \mathbf{f})$ production in the kinematic range specified in Table 1. The cross sections are compared to the leading order, $\mathcal{O}\left(\alpha^{3} \alpha_{s}^{0}\right)$, calculation (more details in the caption of Fig. 6). The photon plus jet sample is additionally compared to a NLO $\left(\alpha^{3} \alpha_{s}\right)$ calculation. The bin averaged NLO cross sections are indicated by the squares

can be explained by the fact that both topologies correspond to the same order $\mathcal{O}\left(\alpha_{s}^{0}\right)$ in perturbative QCD.

In addition, the differential cross sections $\mathrm{d} \sigma / \mathrm{d} E_{\mathrm{T}}^{\gamma}$, $\mathrm{d} \sigma / \mathrm{d} \eta^{\gamma}$ and $\mathrm{d} \sigma / \mathrm{d} Q^{2}$ for the photon plus jet selection are compared to the NLO prediction (Fig. 10 right). On average, the NLO prediction is higher than the LO prediction, in particular at low $Q^{2}$, but is still lower than the data by roughly $35 \%$. The shapes of all three differential cross sections are described well by the NLO prediction.

\section{Conclusions}

The cross section for isolated photon production $e p \rightarrow e \gamma X$ is measured in deep-inelastic scattering at HERA. Photons with a transverse energy in the range $3<E_{\mathrm{T}}^{\gamma}<10 \mathrm{GeV}$ and with pseudorapidity $-1.2<\eta^{\gamma}<1.8$ are measured in
DIS events in the kinematic region $4<Q^{2}<150 \mathrm{GeV}^{2}$, $y>0.05$ and $W_{X}>50 \mathrm{GeV}$. Compared to previous measurements, the range of $Q^{2}$ is extended from $Q^{2}>35 \mathrm{GeV}^{2}$ to $Q^{2}>4 \mathrm{GeV}^{2}$. The cross section receives contributions from photon radiation by the struck quark $(Q Q)$, as well as from wide angle bremsstrahlung of the initial and final electrons $(L L)$ and their interference.

The data are compared to a $\operatorname{LO} \mathcal{O}\left(\alpha^{3} \alpha_{s}^{0}\right)$ calculation which is shown to underestimate the measured cross sections by roughly a factor of two. The prediction is most significantly below the data at low $Q^{2}$. The shapes of the $\mathrm{d} \sigma / \mathrm{d} E_{\mathrm{T}}^{\gamma}$ and $\mathrm{d} \sigma / \mathrm{d} \eta^{\gamma}$ distributions are described reasonably well. The comparison of data to the LO calculation in bins of $\eta^{\gamma}$ show that the difference in normalisation can mainly be attributed to an underestimation of the $Q Q$ contribution. The data are further compared to predictions from the Monte Carlo generators PYTHIA, for the simulation of photons radiated from the quark, and RAPGAP for photons radiated from the electron. The Monte Carlo 
predictions are very similar to the predictions from the LO calculation and also understimate the data, especially at low $Q^{2}$.

Jet production in events with isolated photons is also studied. The cross sections for events with no or at least one hadronic jet are underestimated by the LO prediction by a similar factor as for the inclusive measurement. Again the expectations are furthest below the data at low $Q^{2}$. The total photon plus jet cross section is roughly double the photon plus no-jets cross section as expected from the calculations. The NLO $\mathcal{O}\left(\alpha^{3} \alpha_{s}^{1}\right)$ prediction for photon plus jet is higher than the LO prediction, most significantly at low $Q^{2}$, but still underestimates the data. The NLO calculation describes the shapes of the differential cross sections reasonably well.

Further theoretical investigations are needed to understand the observed discrepancy between the measurements and the predictions, including for instance the calculation of higher order processes.

Acknowledgements. We are grateful to the HERA machine group whose outstanding efforts have made this experiment possible. We thank the engineers and technicians for their work in constructing and maintaining the H1 detector, our funding agencies for financial support, the DESY technical staff for continual assistance and the DESY directorate for support and for the hospitality which they extend to the non DESY members of the collaboration. We would like to thank Aude Gehrmann-De Ridder, Thomas Gehrmann and Eva Poulsen for providing the LO calculations and many helpful discussions and G. Kramer and $\mathrm{H}$. Spiesberger for the NLO calculation.

\section{References}

1. P. Aurenche et al., Phys. Rev. D 73, 094007 (2006) [hep$\mathrm{ph} / 0602133]$

2. J. Gayler, Acta Phys. Pol. B 37, 715 (2006) [hep-ex/ 0511035]

3. Fermilab E706 Collaboration, L. Apanasevich et al., Phys. Rev. D 70, 092009 (2004) [hep-ex/0407011]

4. CDF Collaboration, F. Abe et al., Phys. Rev. Lett. 73, 2662 (1994) [Erratum-ibid. 74, 1891 (1995)]

5. CDF Collaboration, D. Acosta et al., Phys. Rev. D 65 , 112003 (2002) [hep-ex/0201004]

6. CDF Collaboration, D. Acosta et al., Phys. Rev. D 70, 074008 (2004) [hep-ex/0404022]

7. CDF Collaboration, D. Acosta et al., Phys. Rev. Lett. 95, 022003 (2005) [hep-ex/0412050]

8. D0 Collaboration, B. Abbott et al., Phys. Rev. Lett. 84, 2786 (2000) [hep-ex/9912017]

9. D0 Collaboration, V.M. Abazov et al., Phys. Lett. B 639, 151 (2006) [hep-ex/0511054]

10. OPAL Collaboration, P.D. Acton et al., Z. Phys. C 58, 405 (1993)

11. ALEPH Collaboration, D. Buskulic et al., Z. Phys. C 57, 17 (1993)

12. DELPHI Collaboration, P. Abreu et al., Z. Phys. C 53, 555 (1992)

13. L3 Collaboration, O. Adriani et al., Phys. Lett. B 292, 472 (1992)
14. ZEUS Collaboration, J. Breitweg et al., Phys. Lett. B 472, 175 (2000) [hep-ex/9910045]

15. H1 Collaboration, A. Aktas et al., Eur. Phys. J. C 38, 437 (2005) [hep-ex/0407018]

16. ZEUS Collaboration, S. Chekanov et al., Eur. Phys. J. C 49, 511 (2007) [hep-ex/0608028]

17. ZEUS Collaboration, S. Chekanov et al., Phys. Lett. B 595, 86 (2004) [hep-ex/0402019]

18. A. Gehrmann-De Ridder, T. Gehrmann, E. Poulsen, Phys. Rev. Lett. 96, 132002 (2006) [hep-ph/0601073] and private communication

19. A. Gehrmann-De Ridder, T. Gehrmann, E. Poulsen, Eur. Phys. J. C 47, 395 (2006) [hep-ph/0604030]

20. T. Sjöstrand et al., PYTHIA 6.2 Physics and Manual (Version 6.224 is used) [hep-ph/0108264]

21. H. Jung, Comput. Phys. Commun. 86, 147 (1995) (Version 3.1 is used). (see also http://www.desy.de/ jung/ rapgap.html)

22. A. Gehrmann-De Ridder, G. Kramer, H. Spiesberger, Nucl. Phys. B 578, 326 (2000) [hep-ph/0003082] and private communication

23. I. Abt et al., Nucl. Instrum. Methods A 386, 310 (1997)

24. I. Abt et al., Nucl. Instrum. Methods A 386, 348 (1997)

25. H1 Calorimeter Group, B. Andrieu et al., Nucl. Instrum. Methods A 336, 460 (1993)

26. H1 Calorimeter Group, B. Andrieu et al., Nucl. Instrum. Methods A 350, 57 (1994)

27. H1 Calorimeter Group, B. Andrieu et al., Nucl. Instrum. Methods A 336, 499 (1993)

28. R.D. Appuhn et al., Nucl. Instrum. Methods A 386, 397 (1997)

29. T. Wolff et al., Nucl. Instrum. Methods A 323, 537 (1992)

30. A. Baird et al., Nucl. Instrum. Methods A 461, 461 (2001)

31. A. Schöning et al., Nucl. Instrum. Methods A 518, 542 (2004)

32. K. Müller et al., Nucl. Instrum. Methods A 312, 457 (1992)

33. S. Eichenberger et al., Nucl. Instrum. Methods A 323, 532 (1992)

34. J. Becker et al., submitted to Nucl. Instrum. Methods A, physics/0701002

35. M. Bengtsson, T. Sjostrand, Z. Phys. C 37, 465 (1988)

36. B. Andersson et al., Phys. Rep. 97, 31 (1983)

37. T. Sjöstrand, Comput. Phys. Commun. 82, 74 (1994)

38. J. Pumplin et al., JHEP 0207, 012 (2002) [hep-ph/ 0201195]

39. G. Corcella et al., HERWIG 6.5 Release Note 135, 128 (2001) [hep-ph/0210213] (Version 6.505 is used)

40. GEANT 3, R. Brun et al., CERN_DD/EE/84-1

41. H1 Collaboration, C. Adloff et al., Eur. Phys. J. C 21, 33 (2001) [hep-ex/0012053]

42. E.W.N. Glover, A.G. Morgan, Z. Phys. C 62, 311 (1994)

43. ALEPH Collaboration, D. Buskulic et al., Z. Phys. C 69 , 365 (1996)

44. S.D. Ellis, D.E. Soper, Phys. Rev. D 48, 3160 (1993) [hep$\mathrm{ph} / 9305266]$

45. C. Schmitz, Ph.D. Thesis, Zürich University, 2007, in preparation, available at http://www-h1.desy.de/publications/ theses_list.html 
46. S. Bentvelsen et al., Proceedings of the Workshop "Physics at HERA", Vol. 1, ed. by W. Buchmüller, G. Ingelman, DESY (1992) 23

47. C. Hoeger, ibid., 43

48. H1 Collaboration, C. Adloff et al., Eur. Phys. J. C 13, 609 (2000) [hep-ex/9908059]
49. K. Koller et al., Z. Phys. C 6, 131 (1980)

50. M. Glück et al., Phys. Rev. Lett. 73, 388 (1994)

51. OPAL Collaboration, K. Ackerstaff et al., Eur. Phys. J. C 2, 39 (1998) [hep-ex/9708020]

52. L. Bourhis, M. Fontannaz, J.P. Guillet, Eur. Phys. J. C 2, 529 (1998) [hep-ph/9704447] 